\title{
Global Spherically Symmetric Solutions to the Equations of a Viscous Polytropic Ideal Gas in an Exterior Domain
}

\author{
Song Jiang \\ Institut für Angewandte Mathematik, Universität Bonn, Wegelerstrasse 10, D-53115 Bonn, \\ Germany. E-mail: unm204@ibm.rhrz.uni-bonn.de
}

Received: 12 June 1995/Accepted: 25 August 1995

Dedicated to Professor Rolf Leis on the occasion of his 65th birthday

\begin{abstract}
We consider the equations of a viscous polytropic ideal gas in the domain exterior to a ball in $\mathbb{R}^{n}$ ( $n=2$ or 3 ) and prove the global existence of spherically symmetric smooth solutions for (large) initial data with spherical symmetry. The large-time behavior of the solutions is also discussed. To prove the existence we first study an approximate problem in a bounded annular domain and then obtain a priori estimates independent of the boundedness of the annular domain. Letting the diameter of the annular domain tend to infinity, we get a global spherically symmetric solution as the limit.
\end{abstract}

\section{Introduction}

The motion of a viscous polytropic ideal gas in $\mathbb{R}^{n}(n=2$ or 3$)$ is described by the following equations in Eulerian coordinates (cf. $[4,25])$

$$
\begin{aligned}
\frac{\partial \rho}{\partial t}+\operatorname{div}(\rho \mathbf{v}) & =0, \\
\rho\left[\frac{\partial \mathbf{v}}{\partial t}+(\mathbf{v} \cdot \nabla) \mathbf{v}\right] & =\mu \Delta \mathbf{v}+(\lambda+\mu) \nabla(\operatorname{div} \mathbf{v})-R \nabla(\rho \theta), \\
c_{V} \rho\left[\frac{\partial \theta}{\partial t}+(\mathbf{v} \cdot \nabla) \theta\right] & =\kappa_{Q} \Delta \theta-R \rho \theta(\operatorname{div} \mathbf{v})+\lambda(\operatorname{div} \mathbf{v})^{2}+2 \mu D \cdot D .
\end{aligned}
$$

Here $\rho, \theta$, and $\mathbf{v}=\left(v_{1}, \ldots, v_{n}\right)$ are the density, the absolute temperature and the velocity respectively, $R, c_{V}$ and $\kappa_{Q}$ are positive constants; $\lambda$ and $\mu$ are the constant viscosity coefficients, $\mu>0, \lambda+2 \mu / n \geqq 0 ; D=D(\mathbf{v})$ is the deformation tensor,

$$
D_{i j}:=\frac{1}{2}\left(\frac{\partial v_{i}}{\partial x_{j}}+\frac{\partial v_{j}}{\partial x_{i}}\right) \quad \text { and } \quad D \cdot D:=\sum_{i, j=1}^{n} D_{i j}^{2} .
$$

Supported by the SFB 256 of the Deutsche Forschungsgemeinschaft at the University of Bonn. 
Let $\Omega:=\left\{x \in \mathbb{R}^{n}|| x \mid>a\right\} \quad(a>0)$ denote the domain exterior to a ball in $\mathbb{R}^{n}$ ( $n=2$ or 3 ). We shall consider the initial boundary value problem of (1.1) in the region $\{t>0, x \in \Omega\}$ with the following initial and boundary conditions

$$
\begin{gathered}
\rho(x, 0)=\rho^{0}(x), \quad \mathbf{v}(x, 0)=\mathbf{v}^{0}(x), \quad \theta(x, 0)=\theta^{0}(x), \quad x \in \bar{\Omega}, \\
\left.\mathbf{v}\right|_{\partial \Omega}=0,\left.\quad \frac{\partial \theta}{\partial v}\right|_{\partial \Omega}=0, \quad t \geqq 0,
\end{gathered}
$$

where $v$ denotes the exterior normal vector.

The global existence of smooth solutions to initial boundary value problems and the Cauchy problem of (1.1) has been investigated by many authors. In one dimension, it is well known that global smooth solutions exist for smooth (large) initial data (e.g. see $[14,3,20,21,22]$ for initial boundary value problems, $[13,3,11]$ for the Cauchy problem; also cf. [12,7-10] for real gases). In more than one dimension the global existence of smooth solutions has been investigated for general domains only in the case of sufficiently small initial data (e.g. see $[18,19,27]$ for initial boundary value problems, $[16,17]$ for the Cauchy problem; also see the survey article [26]).

For large initial data the global existence of solutions to (1.1) has been studied in the case of a bounded annular domain. Nikolaev [23] in 1983 considered the initial boundary value problem of (1.1) with vanishing velocity and constant temperature on the boundary and proved that for (smooth) spherically symmetric initial data a (smooth) spherically symmetric solution exists globally in time if the initial density and temperature are strictly positive. Recently, Yashima and Benabidallah [28,29] dealt with the case of non-negative initial density and temperature. They showed the global existence of spherically symmetric solutions to (1.1). The boundedness of the domain is essential in $[23,28,29]$.

In this paper we prove the global existence of smooth spherically symmetric solutions to (1.1)-(1.3) in the exterior domain $\Omega$ and study the large-time behavior of the solutions.

The paper is organized as follows: In Sect. 2 we derive the spherically symmetric form of (1.1) and present the main result. In Sect. 3 we consider an approximate problem and prove uniform a priori estimates. The proof of the main result is given in Sect. 4. Finally, the large-time behavior of the solutions is investigated in Sect. 5.

Notation. Let $\alpha \in(0,1)$ and $b_{1}, b_{2} \in \mathbb{R}$ with $b_{1} \leqq b_{2} . C^{\alpha}\left[b_{1}, b_{2}\right]$ denotes the Banach space of functions on $\left[b_{1}, b_{2}\right]$ which are uniformly Hölder continuous with exponent $\alpha$ and $C^{\alpha, \alpha / 2}\left(\bar{G}_{T}\right)$ for the Banach space of functions on $\bar{G}_{T}:=\left[b_{1}, b_{2}\right] \times[0, T]$ which are uniformly Hölder continuous with exponents $\alpha$ in $x$ and $\alpha / 2$ in $t$, and $\|\cdot\|_{C^{\alpha}\left[b_{1}, b_{2}\right]}$ and $\|\cdot\|_{C^{\alpha, \alpha / 2}\left(\bar{G}_{T}\right)}$ are their norms, respectively. We define (see $[6,15]$ )

$$
\begin{gathered}
C^{m+\alpha}\left[b_{1}, b_{2}\right]:=\left\{f \in C^{m}\left[b_{1}, b_{2}\right]\left|\sum_{j=0}^{m} \max _{\left[b_{1}, b_{2}\right]}\right| \partial_{x}^{j} f \mid+\left\|\partial_{x}^{m} f\right\|_{C^{\alpha}\left[b_{1}, b_{2}\right]}<\infty\right\}, \\
C^{1+\alpha,(1+\alpha) / 2}\left(\bar{G}_{T}\right):=\left\{g \mid g, g_{x} \in C^{\alpha, \alpha / 2}\left(\bar{G}_{T}\right)\right\}, \\
C^{2+\alpha, 1+\alpha / 2}\left(\bar{G}_{T}\right):=\left\{g \mid g, g_{x}, g_{t}, g_{x x} \in C^{\alpha, \alpha / 2}\left(\bar{G}_{T}\right)\right\} .
\end{gathered}
$$


Denote $Q_{T}:=(0, \infty) \times(0, T)$ and $Q_{T}^{k}:=(0, k) \times(0, T)(k \in \mathbb{N})$. We use

$$
\begin{gathered}
C_{\mathrm{loc}}^{m+\alpha}[0, \infty):=\left\{f \in C^{m+\alpha}[c, d] \mid \forall[c, d] \subset[0, \infty)\right\}, \\
C_{\mathrm{loc}}^{m+\alpha,(m+\alpha) / 2}\left(\bar{Q}_{T}\right):=\left\{g \in C^{m+\alpha,(m+\alpha) / 2}([c, d] \times[0, T]) \mid \forall[c, d] \subset[0, \infty)\right\}, \\
m=0,1,2 .
\end{gathered}
$$

Let $m \geqq 0$ be an non-negative integer and let $1 \leqq p \leqq \infty$. By $W^{m, p}\left(b_{1}, b_{2}\right)$ we denote the usual Sobolev space defined over $\left(b_{1}, b_{2}\right)$ with norm $\|\cdot\|_{W^{m, p}\left(b_{1}, b_{2}\right)}$ (see $[1]) ; W^{m, 2}\left(b_{1}, b_{2}\right) \equiv H^{m}\left(b_{1}, b_{2}\right)$ with norm $\|\cdot\|_{H^{m}\left(b_{1}, b_{2}\right)}, W^{0, p}\left(b_{1}, b_{2}\right) \equiv L^{p}\left(b_{1}, b_{2}\right)$ with norm $\|\cdot\|_{L^{p}\left(b_{1}, b_{2}\right)}$. For simplicity we also use the following abbreviations:

$L^{p} \equiv L^{p}(0, \infty), \quad H^{m} \equiv H^{m}(0, \infty) ; \quad\|\cdot\|_{L^{p}} \equiv\|\cdot\|_{L^{p}(0, \infty)}, \quad\|\cdot\|_{H^{m}} \equiv\|\cdot\|_{H^{m}(0, \infty)}$.

$(\cdot, \cdot)$ and $\|\cdot\|$ stand for the inner product and the norm in $L^{2}(0, \infty)$ respectively, and $\langle\cdot, \cdot\rangle_{Q_{T}}$ for the inner product in $L^{2}\left(Q_{T}\right) . L^{p}(I, B)$ resp. $\|\cdot\|_{L^{p}(I, B)}$ denotes the space of all strongly measurable, $p^{\text {th }}$-power integrable (essentially bounded if $p=\infty$ ) from $I$ to $B$ resp. its norm, $I \subset \mathbb{R}$ an interval, $B$ a Banach space. For a vector valued function $f=\left(f_{1}, \ldots, f_{m}\right)$ and a normed space $X$ with the norm $\|\cdot\| \|, f \in X$ means that each component of $f$ is in $X$; we put $\left\|\left|\|f\|:=\left\|f_{1}\right\|\right|+\cdots+\right\| f_{m} \|$.

The same letter $C$ will denote various positive constants which do not depend on $k$, but possibly on $T$.

\section{Spherically Symmetric Form and the Main Result}

We first derive the spherically symmetric form of (1.1). Spherically symmetric solutions to (1.1) have the form

$$
v_{i}(x, t)=\frac{x_{i}}{r} v(r, t), \quad i=1, \ldots, n, \quad \rho(x, t)=\rho(r, t), \quad \theta(x, t)=\theta(r, t),
$$

where $x=\left(x_{1}, \ldots, x_{n}\right) \in \mathbb{R}^{n}, r:=|x|$. Assuming that $\rho^{0}(x)=\rho_{0}(r), \mathbf{v}^{0}(x)=x v_{0}(r) /$ $r, \theta^{0}(x)=\theta_{0}(r)$, and denoting $\beta:=\lambda+2 \mu$, we thus reduce the system (1.1)-(1.3) to the following equations for $\rho(r, t), v(r, t)$ and $\theta(r, t)$ of the form:

$$
\begin{gathered}
\partial_{t} \rho+\partial_{r}(\rho v)+\frac{(n-1)}{r} \rho v=0 \\
\rho\left(\partial_{t} v+v \partial_{r} v\right)=\beta\left(\partial_{r}^{2} v+\frac{(n-1)}{r} \partial_{r} v-\frac{(n-1)}{r^{2}} v\right)-R \partial_{r}(\rho v), \quad r \in(a, \infty), t>0 \\
c_{V} \rho\left(\partial_{t} \theta+v \partial_{r} \theta\right)=\kappa_{Q} \partial_{r}^{2} \theta+\kappa_{Q} \frac{(n-1)}{r} \partial_{r} \theta-R \rho \theta\left(\partial_{r} v+\frac{(n-1)}{r} v\right) \\
+\lambda\left(\partial_{r} v+\frac{(n-1)}{r} v\right)^{2}+2 \mu\left(\partial_{r} v\right)^{2}+2 \mu \frac{(n-1)}{r^{2}} v^{2}
\end{gathered}
$$


with the initial and boundary conditions

$$
\begin{gathered}
\rho(r, 0)=\rho_{0}(r), \quad v(r, 0)=v_{0}(r), \quad \theta(r, 0)=\theta_{0}(r), \quad r \in[a, \infty), \\
v(a, t)=0, \quad \theta_{r}(a, t)=0, \quad t \geqq 0 .
\end{gathered}
$$

To investigate the global existence it is convenient to transform the system (2.2) to that in Lagrangian coordinates. The Eulerian coordinates $(r, t)$ are connected to the Lagrangian coordinates $(\xi, t)$ by the relation

$$
r(\xi, t)=r_{0}(\xi)+\int_{0}^{t} \tilde{v}(\xi, \tau) d \tau
$$

where $\tilde{v}(\xi, t):=v(r(\xi, t), t)$ and

$$
r_{0}(\xi):=\eta^{-1}(\xi) ; \quad \eta(r):=\int_{a}^{r} s^{n-1} \rho_{0}(s) d s, \quad r \in[a, \infty) .
$$

Note that if $\rho_{0}(s)>0$ for any $s \in[a, \infty)$ (which is assumed in Theorem 2.1 later), then $\eta$ as a function of $r \in[a, \infty)$ is invertible. Using Eq. $(2.2)_{1},(2.4)$ and the boundary condition $v(a, t)=0$, we obtain

$$
\partial_{t} \int_{a}^{r(\xi, t)} s^{n-1} \rho(s, t) d s=0
$$

which, by integrating with respect to $t$, turns into

$$
\int_{a}^{r(\xi, t)} s^{n-1} \rho(s, t) d s=\int_{a}^{r_{0}(\xi)} s^{n-1} \rho_{0}(s) d s=\eta\left(r_{0}(\xi)\right)=\xi .
$$

Thus, we have under the assumption $\rho(s, t)>0$ for any $s \in[a, \infty)$ and $t \geqq 0$ (which is a posteriori justified by (4.9) in Sect. 4) that $r=a$ iff $\xi=0, r \rightarrow \infty$ iff $\xi \rightarrow \infty$, and

$$
\frac{\partial r(\xi, t)}{\partial \xi}=\left[r(\xi, t)^{n-1} \rho(r(\xi, t), t)\right]^{-1} .
$$

For a function $\varphi(r, t)$ we write $\tilde{\varphi}(\xi, t):=\varphi(r(\xi, t), t)$. By virtue of (2.4) and $(2.8)$,

$$
\begin{aligned}
& \partial_{t} \tilde{\varphi}(\xi, t)=\partial_{t} \varphi(r, t)+v \partial_{r} \varphi(r, t), \\
& \partial_{\xi} \tilde{\varphi}(\xi, t)=\partial_{r} \varphi(r, t) \partial_{\xi} r(\xi, t)=\frac{1}{r^{n-1} \rho(r, t)} \partial_{r} \varphi(r, t) .
\end{aligned}
$$

Without danger of confusion we denote $(\tilde{\rho}, \tilde{v}, \tilde{\theta})$ still by $(\rho, v, \theta)$ and $(\xi, t)$ by $(x, t)$. We use $u:=1 / \rho$ to denote the specific volume. Therefore, by virtue of (2.8)-(2.9), Eqs. (2.2)-(2.3) in the new variables $(x, t)$ read

$$
\begin{gathered}
u_{t}=\left(r^{n-1} v\right)_{x}, \\
v_{t}=r^{n-1}\left[\beta \frac{\left(r^{n-1} v\right)_{x}}{u}-R \frac{\theta}{u}\right]_{x}, \quad x \in(0, \infty), t>0, \\
c_{V} \theta_{t}=\kappa_{Q}\left[\frac{r^{2 n-2} \theta_{x}}{u}\right]_{x}+\frac{1}{u}\left[\beta\left(r^{n-1} v\right)_{x}-R \theta\right]\left(r^{n-1} v\right)_{x}-2 \mu(n-1)\left(r^{n-2} v^{2}\right)_{x}
\end{gathered}
$$


with the initial and boundary conditions

$$
\begin{gathered}
u(x, 0)=u_{0}(x), \quad v(x, 0)=v_{0}(x), \quad \theta(x, 0)=\theta_{0}(x), \quad x \in[0, \infty), \\
v(0, t)=0, \quad \theta_{x}(0, t)=0, \quad t \geqq 0,
\end{gathered}
$$

where $u_{0}:=1 / \rho_{0}, \beta=\lambda+2 \mu$, and by virtue of (2.4)-(2.5), $r \equiv r(x, t)$ is determined by

$$
\begin{aligned}
r(x, t) & =r_{0}(x)+\int_{0}^{t} v(x, \tau) d \tau, \quad x \in[0, L], t \geqq 0, \\
r_{0}(x) & :=\left\{a^{n}+n \int_{0}^{x} u_{0}(y) d y\right\}^{1 / n} .
\end{aligned}
$$

As mentioned in the introduction our aim is to prove a global existence theorem for $(2.10)-(2.14)$. For this purpose we assume that

$$
u_{0}-1, v_{0}, \theta_{0}-1, r_{0}^{n-1} \partial_{x} u_{0}, r_{0}^{n-1} \partial_{x} v_{0}, r_{0}^{n-1} \partial_{x} \theta_{0} \in L^{2}
$$

Before stating the main result we introduce the following definition:

Definition. $\{u, v, \theta\}$ is called a (global) generalized solution of (2.10)-(2.15), if for every $T>0$,

$$
\begin{gathered}
u-1, v, \theta-1 \in L^{\infty}\left([0, T], H^{1}\right), \quad u_{t} \in L^{\infty}\left([0, T], L^{2}\right), \\
v_{t}, \theta_{t}, u_{x t}, v_{x x}, \theta_{x x}, r^{n-1} \theta_{x}, r^{2 n-2} \theta_{x x} \in L^{2}\left(Q_{T}\right),
\end{gathered}
$$

and $\{u, v, \theta\}$ satisfies $(2.10)-(2.15)$ almost everywhere in $Q_{T} \equiv(0, \infty) \times(0, T)$ and takes on the given boundary and initial conditions in the sense of traces.

It should be noted that $r(x, t) \geqq a,(x, t) \in \bar{Q}_{T}$ (cf. (3.19) in Sect. 3), and if $\{u, v, \theta\}$ is a generalized solution, then $u, v, \theta \in C^{0}([0, \infty) \times[0, T])$ (see the proof of (4.8) in Sect. 4). The main result of the paper reads:

Theorem 2.1. Assume that (2.16) is satisfied and $u_{0}(x), \theta_{0}(x)>0$ for $x \in[0, \infty)$. Let the initial data be compatible with the boundary conditions (2.14). Then the problem (2.10)-(2.15) has a unique generalized solution $\{u, v, \theta\}$ with $u, \theta>0$ on $\bar{Q}_{T}$. If in addition

$$
\begin{gathered}
u_{0} \in C_{\mathrm{loc}}^{1+\alpha}[0, \infty), v_{0}, \theta_{0}-1 \in C_{\mathrm{loc}}^{2+\alpha}(0, \infty) \text { for some } \alpha \in(0,1), \\
r_{0}^{2 n-2}\left(v_{0}\right)_{x x}, r_{0}^{2 n-2}\left(\theta_{0}\right)_{x x} \in L^{2}(0, \infty)
\end{gathered}
$$

then

$$
u, u_{t} \in C_{\mathrm{loc}}^{1+\alpha,(1+\alpha) / 2}\left(\bar{Q}_{T}\right), \quad v, \theta \in C_{\mathrm{loc}}^{2+\alpha, 1+\alpha / 2}\left(\bar{Q}_{T}\right) .
$$

The proof of Theorem 2.1 is essentially based on a careful examination of a priori estimates and a limit procedure. Since the domain is unbounded and the coefficients tend to infinity as $x \rightarrow \infty$, some difficulties arise; for example, from the a priori estimates we could get only $v(x, t)=o\left(x^{-1 / 2+1 /(2 n)}\right)$ as $x \rightarrow \infty$; but this is not sufficient to guarantee integration by parts in the proof where $v=o\left(x^{-1+1 / n}\right)$ is required. To overcome such difficulties we first study an approximate problem in the bounded interval $(0, k)$ and show the a priori estimates independent of $k$ by utilizing some cut-off function and modifying a technique of Kazhikhov [13,3] for 
the one-dimensional case, then letting $k$ tend to infinity and using the obtained a priori estimates, we get a global spherically symmetric solution as the limit.

Remark 2.2. The same techniques work and an analogous theorem is obtained when (1.3) is replaced by the following boundary conditions:

$$
\left.\mathbf{v}\right|_{\partial \Omega}=0,\left.\quad \theta\right|_{\partial \Omega}=1, \quad t \geqq 0 .
$$

\section{A Priori Estimates for the Approximate Problem}

As mentioned in the introduction, to show Theorem 2.1 we first consider an approximate problem in $(0, k)(k \in \mathbb{N})$ to $(2.10)-(2.15)$, and prove the uniform a priori estimates for solutions of the approximate problem, then we let $k$ go to infinity to get the existence.

We start with the construction of the approximate initial data $\left\{u_{k}^{0}, v_{k}^{0}, \theta_{k}^{0}\right\}$. For $k \in \mathbb{N}$ let $\phi_{k}$ be a $C^{3}$-function on $\mathbb{R}$ such that $\phi_{k}(x)=1$ for $x \in[0, k / 2]$ and $\phi_{k}(x)=0$ for $x \geqq k$, and

$$
\begin{aligned}
& 0 \leqq \phi_{k}(x) \leqq 1, \quad\left|\phi_{k}^{(i)}(x)\right| \leqq C / k^{i}, \quad i=1,2,3 \\
& \quad \text { for all } x \in[0, \infty) \text { and } k \in \mathbb{N},
\end{aligned}
$$

where $C$ is a positive constant independent of $k$. We define

$$
\begin{aligned}
& u_{k}^{0}(x):=\left(u_{0}(x)-1\right) \phi_{k}(x)+1, \quad v_{k}^{0}(x):=v_{0}(x) \phi_{k}(x), \\
& \theta_{k}^{0}(x):=\left(\theta_{0}(x)-1\right) \phi_{k}(x)+1, \quad x \in[0, \infty) .
\end{aligned}
$$

We consider the following approximate problem to $(2.10)-(2.15)$ in the bounded interval $(0, k)$ :

$$
\begin{aligned}
\partial_{t} u_{k}= & \left(r_{k}^{n-1} v_{k}\right)_{x} \\
\partial_{t} v_{k}= & r_{k}^{n-1}\left[\beta \frac{\left(r_{k}^{n-1} v_{k}\right)_{x}}{u_{k}}-R \frac{\theta_{k}}{u_{k}}\right]_{x}, \\
c_{V} \partial_{t} \theta_{k}= & \kappa_{Q}\left[\frac{r_{k}^{2 n-2}\left(\theta_{k}\right)_{x}}{u_{k}}\right]_{x}+\frac{1}{u_{k}}\left[\beta\left(r_{k}^{n-1} v_{k}\right)_{x}-R \theta_{k}\right]\left(r_{k}^{n-1} v_{k}\right)_{x} \\
& -2 \mu(n-1)\left(r_{k}^{n-2} v_{k}^{2}\right)_{x}, \quad x \in(0, k), t>0,
\end{aligned}
$$

with the initial and boundary conditions

$$
\begin{array}{r}
u_{k}(x, 0)=u_{k}^{0}(x), \quad v_{k}(x, 0)=v_{k}^{0}(x), \quad \theta_{k}(x, 0)=\theta_{k}^{0}(x), \quad x \in[0, k], \\
v_{k}(0, t)=v_{k}(k, t)=0, \quad \partial_{x} \theta_{k}(0, t)=\partial_{x} \theta_{k}(k, t)=0, \quad t \geqq 0,
\end{array}
$$

where $k \in \mathbb{N}$, and

$$
\begin{aligned}
r_{k}(x, t) & =r_{k}^{0}(x)+\int_{0}^{t} v_{k}(x, \tau) d \tau, \quad x \in[0, k], t \geqq 0, \\
r_{k}^{0}(x) & :=\left\{a^{n}+n \int_{0}^{x} u_{k}^{0}(y) d y\right\}^{1 / n} .
\end{aligned}
$$


From the definition of $\left\{u_{k}^{0}, v_{k}^{0}, \theta_{k}^{0}\right\}$ it is easy to see that the initial data $u_{k}^{0}, v_{k}^{0}$, $\theta_{k}^{0}$ are compatible with the boundary conditions (3.7) as $u_{0}, v_{0}, \theta_{0}$ do, and under the conditions of Theorem 2.1 we have

$$
c_{0}^{-1} \leqq u_{k}^{0}(x), \quad \theta_{k}^{0}(x) \leqq c_{0}, \quad\left|v_{k}^{0}(x)\right| \leqq c_{0} \quad \forall x \in[0, k], k \in \mathbb{N}
$$

for some positive constant $c_{0}$ independent of $k$. Moreover, one has

Lemma 3.1. Under the conditions of Theorem 2.1 we have

$$
\begin{aligned}
& \left\{u_{k}^{0}, v_{k}^{0}, \theta_{k}^{0},\left(r_{k}^{0}\right)^{n-1} \partial_{x} u_{k}^{0},\left(r_{k}^{0}\right)^{n-1} \partial_{x} v_{k}^{0},\left(r_{k}^{0}\right)^{n-1} \partial_{x} \theta_{k}^{0}\right\} \\
& \quad \rightarrow\left\{u_{0}, v_{0}, \theta_{0}, r_{0}^{n-1} \partial_{x} u_{0}, r_{0}^{n-1} \partial_{x} v_{0}, r_{0}^{n-1} \partial_{x} \theta_{0}\right\} \quad \text { in } L^{2}(0, \infty) \text { as } k \rightarrow \infty
\end{aligned}
$$

If in addition (2.18) holds, then

$$
\begin{aligned}
\left\{\left(r_{k}^{0}\right)^{2 n-2} \partial_{x}^{2} v_{k}^{0},\left(r_{k}^{0}\right)^{2 n-2} \partial_{x}^{2} \theta_{k}^{0}\right\} \rightarrow & \left\{r_{0}^{2 n-2} \partial_{x}^{2} v_{0}, r_{0}^{2 n-2} \partial_{x}^{2} \theta_{0}\right\} \\
& \text { in } L^{2}(0, \infty) \text { as } k \rightarrow \infty
\end{aligned}
$$

Proof. In view of (2.16) we easily see that $\left\|\left(u_{k}^{0}-u_{0}, v_{k}^{0}-v_{0}, \theta_{k}^{0}-\theta_{0}\right)\right\| \rightarrow 0$ as $k \rightarrow \infty$. From (3.9) and the definition of $r_{0}$ and $r_{k}^{0}$ one gets

$$
C^{-1}\left(1+x^{1 / n}\right) \leqq r_{0}(x), r_{k}^{0}(x) \leqq C\left(1+x^{1 / n}\right)
$$

$$
\text { for all } x \in[0, \infty) \text { and } k \in \mathbb{N} \text {, }
$$

which together with (3.1) and Cauchy-Schwarz's inequality implies

$$
\begin{gathered}
\left\|\left(r_{k}^{0}\right)^{n-1} \phi_{k}^{\prime}\right\|_{L^{\infty}} \leqq C k^{-1 / n} \quad \forall k \geqq 1, \\
\left\|\left[\left(r_{k}^{0}\right)^{n-1}-r_{0}^{n-1}\right] r_{0}^{-n+1}\right\|_{L^{\infty}} \leqq C\left\|\left[\left(r_{k}^{0}\right)^{n}-r_{0}^{n}\right] r_{0}^{-n+1}\right\|_{L^{\infty}} \\
\leqq C \sup _{x \geqq 0}\left|r_{0}^{-n+1}(x) \int_{0}^{x}\left(1-\phi_{k}\right)\left(1-u_{0}\right) d y\right| \leqq C \sup _{x \geqq k / 2} x^{-1+1 / n} \int_{k / 2}^{x}\left|1-u_{0}\right| d y \\
\leqq C\left\|1-u_{0}\right\|_{L^{2}(k / 2, \infty)} \rightarrow 0 \quad \text { as } k \rightarrow \infty .
\end{gathered}
$$

Hence from (3.12)-(3.13) and (2.16), it follows that

$$
\begin{aligned}
& \left\|\left(r_{k}^{0}\right)^{n-1}\left(v_{k}^{0}\right)_{x}-r_{0}^{n-1}\left(v_{0}\right)_{x}\right\| \leqq\left\|\left(r_{k}^{0}\right)^{n-1} \phi_{k}^{\prime} v_{0}\right\|+\left\|\left[\left(r_{k}^{0}\right)^{n-1} \phi_{k}-r_{0}^{n-1}\right]\left(v_{0}\right)_{x}\right\| \\
& \quad \leqq C k^{-1 / n}\left\|v_{0}\right\|+\left\|\left(r_{k}^{0}\right)^{n-1}\left(\phi_{k}-1\right)\left(v_{0}\right)_{x}\right\|+\left\|\left[\left(r_{k}^{0}\right)^{n-1}-r_{0}^{n-1}\right]\left(v_{0}\right)_{x}\right\| \\
& \quad \leqq C\left\{k^{-1 / n}+\left\|r_{0}^{n-1}\left(v_{0}\right)_{x}\right\|_{L^{2}(k / 2, \infty)}+\left\|\left[\left(r_{k}^{0}\right)^{n-1}-r_{0}^{n-1}\right] r_{0}^{-n+1}\right\|_{L^{\infty}}\right\} \\
& \quad \rightarrow 0 \quad \text { as } k \rightarrow \infty
\end{aligned}
$$

The rest of (3.10) as well as (3.11) can be shown in the same manner. The proof is complete.

By virtue of the well-known global existence theorem for the problem (3.3)(3.8) (in bounded domains), (3.9) and Lemma 3.1 imply (see [23, 28, 29]). 
Lemma 3.2. Under the conditions of Theorem 2.1 there exists a unique solution $\left\{u_{k}, v_{k}, \theta_{k}\right\}$ with positive $u_{k}, \theta_{k}$ to $(3.3)-(3.8)$ on $[0, k] \times[0, \infty)$ such that for every $T>0$,

$$
\begin{gathered}
u_{k}, v_{k}, \theta_{k} \in L^{\infty}\left([0, T], H^{1}(0, k)\right), \\
\left(u_{k}\right)_{t},\left(v_{k}\right)_{t},\left(\theta_{k}\right)_{t},\left(u_{k}\right)_{x t},\left(v_{k}\right)_{x x},\left(\theta_{k}\right)_{x x} \in L^{2}\left(Q_{T}^{k}\right),
\end{gathered}
$$

where $Q_{T}^{k} \equiv(0, k) \times(0, T)$. If in addition (2.18) holds, and $u_{0}, v_{0}, \theta_{0}$ are compatible with the boundary conditions (2.14), then

$$
u_{k},\left(u_{k}\right)_{t} \in C^{1+\alpha,(1+\alpha) / 2}\left(\bar{Q}_{T}^{k}\right), \quad v_{k}, \theta_{k} \in C^{2+\alpha, 1+\alpha / 2}\left(\bar{Q}_{T}^{k}\right) .
$$

In this section we show that the global solution $\left\{u_{k}, v_{k}, \theta_{k}\right\}$ of (3.3)-(3.8) can be uniformly bounded with respect to $k$, exploiting some relations associated with the second law of thermodynamics and using the (weighted) energy method. The main result of this section reads:

Theorem 3.3. Let the conditions of Theorem 2.1 be satisfied. Then we have for every $T>0$,

$$
\begin{aligned}
& \left\|\left(u_{k}-1, v_{k}, \theta_{k}-1\right)\right\|_{L^{\infty}\left([0, T], H^{1}(0, k)\right)}+\left\|\left(u_{k}\right)_{t}\right\|_{L^{\infty}\left([0, T], L^{2}(0, k)\right)} \\
& \quad+\left\|\left(\left(v_{k}\right)_{t},\left(\theta_{k}\right)_{t},\left(u_{k}\right)_{x t},\left(v_{k}\right)_{x x},\left(\theta_{k}\right)_{x x}\right)\right\|_{L^{2}\left(Q_{T}^{k}\right)} \leqq C \quad \forall k \in \mathbb{N} .
\end{aligned}
$$

If in addition (2.18) holds, then

$$
\begin{aligned}
& \left\|\left(\left(v_{k}\right)_{t},\left(\theta_{k}\right)_{t},\left(u_{k}\right)_{x t},\left(v_{k}\right)_{x x},\left(\theta_{k}\right)_{x x}\right)\right\|_{L^{\infty}\left([0, T], L^{2}(0, k)\right)} \\
& \quad+\left\|\left(\left(u_{k}\right)_{t t},\left(v_{k}\right)_{x t},\left(\theta_{k}\right)_{x t}\right)\right\|_{L^{2}\left(Q_{T}^{k}\right)} \leqq C \quad \forall k \in \mathbb{N} .
\end{aligned}
$$

The proof of Theorem 3.3 is broken up into a sequence of lemmas. For simplicity we will generally suppress the subscript $k$ (and denote $\left(u_{k}, v_{k}, \theta_{k}, r_{k}\right)$ by $(u, v, \theta, r))$ in the calculations of this section that follow. We start with the following identities:

$$
r_{t}(x, t)=v(x, t), \quad r^{n-1}(x, t) r_{x}(x, t)=u(x, t), \quad x \in[0, k], t \geqq 0,
$$

which can easily be verified. In fact, by virtue of (3.3) and (3.8), $r_{t}(x, t)=v(x, t)$ and $\left(r^{0}\right)^{n-1}\left(r^{0}\right)_{x}=u^{0}$. So we get from (3.3) that

$$
\left[r^{n-1}(x, t) r_{x}(x, t)-u(x, t)\right]_{t}=(n-1) r^{n-1} v r_{x}+r^{n-1} v_{x}-\left(r^{n-1} v\right)_{x}=0,
$$

which yields (3.18).

It follows from (3.18) and (3.7)-(3.8) that $r_{x}(0, t)=a^{1-n} u(0, t)>0$ for $t \geqq 0$. If $r_{x}(x, t)>0$ is violated on $[0, k] \times[0, \infty)$, then there are $y \in(0, k]$ and $\tau \in[0, \infty)$ such that $r_{x}(x, t)>0$ for $0 \leqq x<y, 0 \leqq t \leqq \tau$, but $r_{x}(y, \tau)=0$. So by continuity, $r_{x}(x, t) \geqq 0$ for $x \in[0, y]$ and $t \in[0, \tau]$, and we have $r(y, \tau) \geqq r(0, \tau)=a>0$. From (3.18) we get $0=r_{x}(y, \tau)=r^{1-n}(y, \tau) u(y, \tau)>0$, which is a contradiction. This shows $r_{x}(x, t)>0$ for $x \in[0, k], t \geqq 0$. Therefore

$$
r(x, t) \geqq r(0, t)=a>0 \quad \text { for } x \in[0, k], t \geqq 0 .
$$

The following lemma is motivated by the second law of thermodynamics and embodies the dissipative effects of viscosity and thermal diffusion. 
Lemma 3.4. There is a positive constant $e_{0}$, independent of $k$ and $t$, such that

$$
\begin{aligned}
& \int_{0}^{k} U(x, t) d x+\int_{0}^{t} \int_{0}^{k}\left[\kappa_{Q} \frac{r^{2 n-2} \theta_{x}^{2}}{u \theta^{2}}+\left(\lambda+\frac{2 \mu}{n}\right) \frac{\left(r^{n-1} v\right)_{x}^{2}}{u \theta}\right] d x d s \leqq e_{0}, \\
& \forall t \geqq 0, k \in \mathbb{N}
\end{aligned}
$$

where

$$
U(x, t):=\left\{v^{2} / 2+R(u-\log u-1)+c_{V}(\theta-\log \theta-1)\right\}(x, t) .
$$

Proof. Using (3.3)-(3.5), we obtain after a straightforward calculation that

$$
\begin{aligned}
& U_{t}+\frac{\beta}{u \theta}\left(r^{n-1} v\right)_{x}^{2}+\frac{\kappa_{Q}}{u \theta^{2}}\left(r^{n-1} \theta_{x}\right)^{2}=\left[r^{n-1} v\left(\frac{\beta}{u}\left(r^{n-1} v\right)_{x}-R \frac{\theta}{u}\right)\right]_{x}+R\left(r^{n-1} v\right)_{x} \\
& +\kappa_{Q}\left[\left(1-\frac{1}{\theta}\right) \frac{r^{2 n-2} \theta_{x}}{u}\right]_{x}-2 \mu(n-1)\left(1-\frac{1}{\theta}\right)\left(r^{n-2} v^{2}\right)_{x} .
\end{aligned}
$$

If we integrate the above identity over $[0, k] \times[0, t](t \geqq 0)$ and use the boundary conditions (3.7), we obtain

$$
\begin{aligned}
& \int_{0}^{k} U(x, t) d x+\beta \int_{0}^{t} \int_{0}^{k} \frac{\left(r^{n-1} v\right)_{x}^{2}}{u \theta} d x d s+\kappa_{Q} \int_{0}^{t} \int_{0}^{k} \frac{r^{2 n-2} \theta_{x}^{2}}{u \theta^{2}} d x d s \\
& =\int_{0}^{k} U(x, 0) d x+2 \mu(n-1) \int_{0}^{t} \int_{0}^{k} \frac{\left(r^{n-2} v^{2}\right)_{x}}{\theta} d x d s, \quad t \geqq 0 .
\end{aligned}
$$

Recalling $n \lambda+2 \mu \geqq 0$, we make use of (3.18) to deduce

$$
\begin{aligned}
& \frac{\beta}{u}\left(r^{n-1} v\right)_{x}^{2}-2 \mu(n-1)\left(r^{n-2} v^{2}\right)_{x}=\left(\lambda+\frac{2}{n} \mu\right) \frac{\left(r^{n-1} v\right)_{x}^{2}}{u} \\
& \quad+\frac{2 \mu(n-1)}{u}\left\{\frac{\left(r^{n-1} v\right)_{x}}{\sqrt{n}}-\frac{\sqrt{n} u v}{r}\right\}^{2} \geqq 0 .
\end{aligned}
$$

By virtue of Taylor's theorem and (3.9)-(3.10), $\int_{0}^{k} U(x, 0) d x \leqq C\left(1+\|\left(u_{0}-\right.\right.$ $\left.\left.1, v_{0}, \theta_{0}-1\right) \|^{2}\right)(\forall k \geqq 1)$; so substituting (3.23) into (3.22), we obtain (3.20). The proof is complete.

From Lemma 3.4 we see that

$$
\begin{gathered}
\int_{i}^{i+1}(u-\log u-1)(x, t) d x \\
\int_{i}^{i+1}(\theta-\log \theta-1)(x, t) d x \leqq e_{0} / \min \left\{R, c_{V}\right\}, \quad i=0, \ldots, k-1 .
\end{gathered}
$$

Hence by terms of the mean value theorem, for each $t \geqq 0$ there are points $a_{i}(t), b_{i}(t) \in[i, i+1](i=0, \ldots, k-1)$ such that

$$
0<\alpha_{1} \leqq u\left(a_{i}(t), t\right), \theta\left(b_{i}(t), t\right) \leqq \alpha_{2}, \quad t \geqq 0, \quad i=0, \ldots, k-1,
$$


where $\alpha_{1}, \alpha_{2}$ are two (positive) roots of the equation $y-\log y-1=e_{0} / \min \left\{R, c_{V}\right\}$. Moreover, if we utilize (3.24) and apply Jensen's inequality to the convex function $y-\log y-1$, we obtain:

$$
\begin{gathered}
\int_{i}^{i+1} u(x, t) d x-\log \int_{i}^{i+1} u(x, t) d x-1, \\
\int_{i}^{i+1} \theta(x, t) d x-\log \int_{i}^{i+1} \theta(x, t) d x-1 \leqq e_{0} / \min \left\{R, c_{V}\right\},
\end{gathered}
$$

which gives $\alpha_{1} \leqq \int_{i}^{i+1} u(x, t) d x, \int_{i}^{i+1} \theta(x, t) d x \leqq \alpha_{2}$ for $i=0, \ldots, k-1$. In view of (3.25), we thus have proved

\section{Lemma 3.5.}

$$
\alpha_{1} \leqq \int_{i}^{i+1} u(x, t) d x, \quad \int_{i}^{i+1} \theta(x, t) d x \leqq \alpha_{2} \quad t \geqq 0, i=0, \ldots, k-1,
$$

and for each $t \geqq 0$ there are points $a_{i}(t), b_{i}(t) \in[i, i+1](i=0, \ldots, k-1)$ such that

$$
\alpha_{1} \leqq u\left(a_{i}(t), t\right), \quad \theta\left(b_{i}(t), t\right) \leqq \alpha_{2}, \quad t \geqq 0, i=0, \ldots, k-1
$$

where $\alpha_{1}, \alpha_{2}$ are two (positive) roots of the equation $y-\log y-1=e_{0} / \min \left\{R, c_{V}\right\}$ and the constant $e_{0}$ is the same as in Lemma 3.4.

Our next object is to derive pointwise bounds on the specific volume $u$. We have

Lemma 3.6. There are positive constants $\underline{u}$ and $\bar{u}$, independent of $k$, such that

$$
\underline{u} \leqq u(x, t) \leqq \bar{u} \text { for any } x \in[0, k], t \in[0, T] .
$$

Proof. Using (3.3)-(3.4), we rewrite (3.4) as follows:

$$
\frac{1}{\beta} r^{-n+1} v_{t}+\frac{R}{\beta}\left[\frac{\theta}{u}\right]_{x}=[\log u]_{x t}
$$

Integrating (3.29) over $[0, t]$ with respect to $t$, then over $\left[a_{i}(t), x\right]$ with respect to $x$ for $x \in[i, i+1](0 \leqq i \leqq k-1)$, we obtain

$$
\begin{aligned}
\frac{R}{\beta} \int_{0}^{t} \frac{\theta(x, s)}{u(x, s)} d s-\log u(x, t)= & \log \frac{u^{0}\left(a_{i}(t)\right)}{u^{0}(x)}-\log u\left(a_{i}(t), t\right) \\
& +\frac{R}{\beta} \int_{0}^{t} \frac{\theta\left(a_{i}(t), s\right)}{u\left(a_{i}(t), s\right)} d s-\frac{1}{\beta} \int_{a_{i}(t)}^{x} \int_{0}^{t} r^{-n+1} v_{t} d y d s,
\end{aligned}
$$

which, when the exponentials are taken, turns into

$$
\begin{gathered}
\frac{1}{u(x, t)} \exp \left\{\frac{R}{\beta} \int_{0}^{t} \frac{\theta(x, s)}{u(x, s)} d s\right\}=\frac{1}{u\left(a_{i}(t), t\right)} Y_{i}(t) B_{i}(x, t), \quad t \in[0, T], \\
x \in[i, i+1], 0 \leqq i \leqq k-1,
\end{gathered}
$$


where (noting $u, \theta>0$ )

$$
\begin{gathered}
Y_{i}(t):=\exp \left\{\frac{R}{\beta} \int_{0}^{t} \frac{\theta\left(a_{i}(t), s\right)}{u\left(a_{i}(t), s\right)} d s\right\} \geqq 1, \\
B_{i}(x, t):=\frac{u^{0}\left(a_{i}(t)\right)}{u^{0}(x)} \exp \left\{-\frac{1}{\beta} \int_{a_{i}(t)}^{x} \int_{0}^{t} r^{-n+1} v_{t} d y d s\right\} \geqq 0 .
\end{gathered}
$$

Multiplying (3.30) by $R \theta / \beta$ and integrating over $[0, t](t \in[0, T])$ in the variable $t$, we arrive at

$$
\exp \left\{\frac{R}{\beta} \int_{0}^{t} \frac{\theta(x, s)}{u(x, s)} d s\right\}=1+\frac{R}{\beta} \int_{0}^{t} \frac{\theta(x, s)}{u\left(a_{i}(s), s\right)} Y_{i}(s) B_{i}(x, s) d s .
$$

Inserting (3.33) into (3.30) and utilizing (3.27), one gets for $t \in[0, T], x \in[i, i+1]$, $i=0, \ldots, k-1$,

$$
\begin{aligned}
u(x, t) Y_{i}(t) B_{i}(x, t) & =u\left(a_{i}(t), t\right)\left(1+\frac{R}{\beta} \int_{0}^{t} \frac{\theta(x, s)}{u\left(a_{i}(s), s\right)} Y_{i}(s) B_{i}(x, s) d s\right) \\
& \leqq C\left(1+\int_{0}^{t} \theta(x, s) Y_{i}(s) B_{i}(x, s) d s\right) .
\end{aligned}
$$

If we integrate by parts with respect to $t$, use (3.18)-(3.19), Cauchy-Schwarz's inequality, and (3.20), (3.10), we infer

$$
\begin{aligned}
& \left|\int_{a_{l}(t)}^{x} \int_{0}^{t} r^{-n+1} v_{t} d y d s\right|=\left|\int_{a_{i}(t)}^{x} \int_{0}^{t} \frac{d}{d t}\left(r^{-n+1} v\right) d y d s+(n-1) \int_{a_{i}(t)}^{x} \int_{0}^{t} r^{-n} v^{2} d y d s\right| \\
& \quad \leqq\left|\int_{a_{t}(t)}^{x} r^{-n+1} v d y\right|+\left|\int_{a_{i}(t)}^{x}\left(r^{0}\right)^{-n+1} v^{0} d y\right|+(n-1)\left|\int_{a_{i}(t)}^{x} \int_{0}^{t} r^{-n} v^{2} d y d s\right| \\
& \quad \leqq C\left\{\int_{i}^{i+1} v^{2}(y, t) d y+\int_{i}^{i+1}\left(v^{0}\right)^{2}(y) d y+\int_{0}^{t} \int_{i}^{i+1} v^{2} d y d s\right\} \\
& \quad \leqq C, \quad \forall t \in[0, T], x \in[i, i+1], 0 \leqq i \leqq k-1 .
\end{aligned}
$$

Recalling the definition of $B_{i}(x, t)$, taking (3.9), and (3.35) into account, we see that

$$
0<C^{-1} \leqq B_{i}(x, t) \leqq C \quad \forall t \in[0, T], x \in[i, i+1], 0 \leqq i \leqq k-1 .
$$

Now we integrate (3.34) over $[i, i+1]$ in the variable $x$, employ (3.26) and (3.36) to arrive at $Y_{i}(t) \leqq C\left(1+\int_{0}^{t} Y_{i}(s) d s\right)$ for $t \in[0, T]$ and $0 \leqq i \leqq k-1$. This together with Gronwall's inequality leads to $Y_{i}(t) \leqq C$ for all $t \in[0, T]$ and $0 \leqq i \leqq k-1$, from which, and (3.30), (3.27), (3.36), (3.34), and (3.31), one gets

$$
\begin{gathered}
\underline{u} \leqq u(x, t) \leqq C\left(1+\int_{0}^{t} \max _{[i, i+1]} \theta(\cdot, s) d s\right), \\
t \in[0, T], x \in[i, i+1], 0 \leqq i \leqq k-1,
\end{gathered}
$$

where $\underline{u}$ is a positive constant independent of $i$ and $k$. 
It follows from Cauchy-Schwarz's inequality and (3.26) that for all $x \in[i, i+1]$,

$$
\begin{aligned}
\left|\theta^{1 / 2}(x, t)-\theta^{1 / 2}\left(b_{i}(t), t\right)\right| & \leqq \int_{b_{i}(t)}^{x} \theta^{-1 / 2}\left|\theta_{x}\right| d y \leqq\left(\int_{i}^{i+1} \frac{\theta_{x}^{2}}{u \theta^{2}} d y\right)^{1 / 2}\left(\int_{i}^{i+1} u \theta d y\right)^{1 / 2} \\
& \leqq \sqrt{\alpha_{2}}\left(\int_{i}^{i+1} \frac{\theta_{x}^{2}}{u \theta^{2}} d y\right)^{1 / 2} \max _{[i, i+1]} u^{1 / 2}(\cdot, t)
\end{aligned}
$$

Substitution of (3.38) into (3.37) and use of (3.27) yield immediately

$$
\max _{[i, i+1]} u(\cdot, t) \leqq C+C \int_{0}^{t} \int_{i}^{i+1} \frac{\theta_{x}^{2}}{u \theta^{2}} d y \max _{[i, i+1]} u_{k}(\cdot, s) d s
$$

Applying Gronwall's inequality to (3.39) and using (3.19)-(3.20), we see that for any $0 \leqq i \leqq k-1$ and $t \in[0, T] \max _{[i, i+1]} u(\cdot, t) \leqq \bar{u}$ with $\bar{u}$ being a positive constant independent of $i$ and $k$. This together with (3.37) proves the lemma.

Next we apply the energy method to derive Sobolev-norm estimates of derivatives for $u, v, \theta$.

Denote (recall (3.18))

$$
\begin{aligned}
& \sigma(x, t):=\frac{\beta}{u}\left(r^{n-1} v\right)_{x}-R \frac{\theta}{u}=\frac{\beta}{u}\left((n-1) r^{-1} u v+r^{n-1} v_{x}\right)-R \frac{\theta}{u}, \\
& w(x, t):=c_{V}(\theta-1)+v^{2} / 2 .
\end{aligned}
$$

Using (3.3)-(3.4), we may write (3.5) in the form

$$
w_{t}=\left[\sigma r^{n-1} v\right]_{x}+\kappa_{Q}\left[\frac{r^{2 n-2} \theta_{x}}{u}\right]_{x}-2 \mu(n-1)\left[r^{n-2} v^{2}\right]_{x}
$$

Multiply (3.41) by $w$ and integrate. Recalling the boundary conditions (3.7), integrating by parts in the variable $x$, and making use of (3.10), (3.40) 1 , and CauchySchwarz's inequality, (3.19), and (3.28), we obtain

$$
\begin{aligned}
& \frac{1}{2} \int_{0}^{k} w^{2}(x, t) d x \\
& \quad=\frac{1}{2} \int_{0}^{k} w^{2}(x, 0) d x-\int_{0}^{t} \int_{0}^{k}\left\{\sigma r^{n-1} v+\kappa_{Q} \frac{r^{2 n-2} \theta_{x}}{u}-2 \mu(n-1) r^{n-2} v^{2}\right\} w_{x} d x d s \\
& \quad \leqq C-\frac{c_{V} \kappa_{Q}}{4} \int_{0}^{t} \int_{0}^{k} \frac{r^{2 n-2} \theta_{x}^{2}}{u}+C \int_{0}^{t} \int_{0}^{k}\left\{r^{2 n-2} v^{2} v_{x}^{2}+v^{4}+\theta^{2} v^{2}\right\} d x d s
\end{aligned}
$$


for $t \in[0, T]$. To bound the term $\int_{0}^{t} \int_{0}^{k} r^{2 n-2} v^{2} v_{x}^{2} d x d s$, we multiply (3.4) by $v^{3}$ and integrate over $[0, k] \times[0, t]$ to arrive at

$$
\begin{aligned}
\frac{1}{4} \int_{0}^{k} v^{4}(x, t) d x= & \frac{1}{4} \int_{0}^{k}\left(v^{0}\right)^{4} d x-\int_{0}^{t} \int_{0}^{k} \sigma(x, s)\left(r^{n-1} v^{3}\right)_{x} d x d s \\
= & \frac{1}{4} \int_{0}^{k}\left(v^{0}\right)^{4} d x-\int_{0}^{t} \int_{0}^{k}\left\{\beta\left[(n-1) \frac{v}{r}+\frac{r^{n-1} v_{x}}{u}\right]-R \frac{\theta}{u}\right\} \\
& \times\left\{3 r^{n-1} v^{2} v_{x}+(n-1) r^{-1} u v^{3}\right\} d x d s \\
\leqq & C-\frac{\beta}{\bar{u}} \int_{0}^{t} \int_{0}^{k} r^{2 n-2} v^{2} v_{x}^{2} d x d s+C \int_{0}^{t} \int_{0}^{k}\left(v^{4}+v^{2} \theta^{2}\right) d x d s,
\end{aligned}
$$

where we have used (3.10), (3.18)-(3.19), and (3.28). Note that $w^{2}+v^{4} \geqq$ $\frac{1}{2}\left(c_{V}^{2}(\theta-1)^{2}+v^{4}\right)$. So we multiply $(3.43)$ by $(2 \bar{u} C) / \beta$ and add the resulting inequality to (3.42), and utilize (3.28) and (3.20), we infer

$$
\begin{aligned}
& \int_{0}^{k}\left\{(\theta-1)^{2}+v^{4}\right\}(x, t) d x+\int_{0}^{t} \int_{0}^{k} r^{2 n-2}\left(\theta_{x}^{2}+v^{2} v_{x}^{2}\right) d x d s \\
& \leqq C+C \int_{0}^{t} \int_{0}^{k}\left(v^{4}+v^{2} \theta^{2}\right) d x d s \\
& \leqq C+C \int_{0}^{t} \int_{0}^{k} v^{4} d x d s+C \int_{0}^{t} \max _{[0, k]} \theta^{2}(\cdot, s) d s
\end{aligned}
$$

On the other hand, it follows from (3.20) that for any $x \in[i, i+1](0 \leqq i \leqq k-1)$,

$$
\begin{aligned}
\left(\theta(x, t)-\theta\left(b_{i}(t), t\right)\right)^{2} & =\left(\int_{b_{i}(t)}^{x} \theta_{x} d y\right)^{2} \leqq \int_{i}^{i+1} \frac{\theta_{x}^{2}}{\theta^{2}} d y \int_{i}^{i+1} \theta^{2} d y \\
& \leqq 2 \int_{i}^{i+1} \frac{\theta_{x}^{2}}{\theta^{2}} d y \int_{i}^{i+1}(\theta-1)^{2} d y+2 \int_{i}^{i+1} \frac{\theta_{x}^{2}}{\theta^{2}} d y .
\end{aligned}
$$

Therefore by (3.27) and (3.19),

$$
\begin{aligned}
\max _{[i, i+1]} \theta^{2}(\cdot, t) & \leqq 2 \max _{[i, i+1]}\left(\theta(\cdot, t)-\theta\left(b_{i}(t), t\right)\right)^{2}+2 \alpha_{2}^{2} \\
& \leqq 2 \alpha_{2}^{2}+4 \int_{0}^{k} \frac{\theta_{x}^{2}}{\theta^{2}} d x \int_{0}^{k}(\theta-1)^{2} d x+4 \int_{0}^{k} \frac{\theta_{x}^{2}}{\theta^{2}} d x
\end{aligned}
$$

for all $t \in[0, T]$ and $0 \leqq i \leqq k-1$. In view of (3.19)-(3.20) and (3.28), (3.45) implies

$$
\begin{gathered}
\int_{0}^{t} \max _{[i, i+1]} \theta^{2}(\cdot, s) d s \leqq 2 \alpha_{2}^{2} T+4 \int_{0}^{t} \int_{0}^{k} \frac{\theta_{x}^{2}}{\theta^{2}} d x \int_{0}^{k}(\theta-1)^{2} d x d s+4 \int_{0}^{t} \int_{0}^{k} \frac{\theta_{x}^{2}}{\theta^{2}} d x d s \\
\leqq C+C \int_{0}^{t} \int_{0}^{k} \frac{r^{2 n-2} \theta_{x}^{2}}{u \theta^{2}} d x \int_{0}^{k}(\theta-1)^{2} d x d s, \quad t \in[0, T]
\end{gathered}
$$


Inserting (3.46) into (3.44), applying Gronwall's inequality and (3.20), we obtain immediately

Lemma 3.7. For all $t \in[0, T]$ we have

$$
\begin{aligned}
& \int_{0}^{k}\left\{(\theta-1)^{2}+v^{4}\right\}(x, t) d x+\int_{0}^{t} \int_{0}^{k} r^{2 n-2}\left\{\theta_{x}^{2}+v^{2} v_{x}^{2}\right\} d x d s \\
& \quad+\int_{0}^{t} \max _{[0, k]} \theta^{2}(\cdot, s) d s \leqq C .
\end{aligned}
$$

As a consequence of Lemma 3.7 we have

\section{Lemma 3.8.}

$$
\int_{0}^{t} \int_{0}^{k} r^{2 n-2} v_{x}^{2} d x d s \leqq C \quad \forall t \in[0, T]
$$

Proof. We first observe that (3.3) can be written as $(u-1)_{t}=\left(r^{n-1} v\right)_{x}$. Integrating this over $[0, k]$ and recalling the boundary conditions (3.7), we conclude

$$
\int_{0}^{k}(u(x, t)-1)_{t} d x=0, \quad 0 \leqq t \leqq T
$$

Now multiply (3.4) by $v$ and integrate over $[0, k] \times[0, t](0 \leqq t \leqq T)$. If we integrate by parts with respect to $x$, utilize (3.28), (3.3), and Cauchy-Schwarz's inequality, (3.47), and (3.49), we obtain

$$
\begin{aligned}
& \frac{\lambda+2 \mu}{\bar{u}} \int_{0}^{t} \int_{0}^{k}\left(r^{n-1} v\right)_{x}^{2} d x d s \leqq C+R \int_{0}^{t} \int_{0}^{k} \frac{\theta}{u}\left(r^{n-1} v\right)_{x} d x d s \\
& =C+R \int_{0}^{t} \int_{0}^{k} \frac{(\theta-1)}{u}\left(r^{n-1} v\right)_{x} d x d s+R \int_{0}^{t} \int_{0}^{k} \frac{u_{t}}{u} d x d s \\
& \leqq C+\frac{\beta}{2 \bar{u}} \int_{0}^{t} \int_{0}^{k}\left(r^{n-1} v\right)_{x}^{2} d x d s+R \int_{0}^{t} \int_{0}^{k}(\log u(x, t)-u(x, t)+1)_{t} d x
\end{aligned}
$$

from which, and the fact that $\log u-u+1 \leqq 0$, Taylor's theorem and (3.9)-(3.10), it follows that

$$
\begin{aligned}
\int_{0}^{t} \int_{0}^{k}\left(r^{n-1} v\right)_{x}^{2} d x d s & \leqq C+C \int_{0}^{k}\left(u^{0}-\log u^{0}-1\right)(x) d x \\
& \leqq C+C \int_{0}^{k}\left(u^{0}-1\right)^{2} d x \leqq C, \quad t \in[0, T]
\end{aligned}
$$

By virtue of (3.18)-(3.19) and (3.28), $\left(r^{n-1} v\right)_{x}^{2} \geqq \frac{1}{2} r^{2 n-2} v_{x}^{2}-C v^{2}$. Inserting this into (3.50) and using (3.20), we obtain (3.48). This completes the proof of the lemma. 
Lemma 3.8 together with (3.19)-(3.20) gives immediately

$$
\begin{aligned}
\int_{0}^{t} \max _{[0, k]} v^{2}(\cdot, s) d s & \leqq 2 \int_{0}^{t} \int_{0}^{k}\left|v v_{x}\right| d x d s \\
& \leqq C \int_{0}^{t} \int_{0}^{k}\left(v^{2}+r^{2 n-2} v_{x}^{2}\right) d x d s \leqq C, \quad 0 \leqq t \leqq T .
\end{aligned}
$$

\section{Lemma 3.9.}

$$
\int_{0}^{k} r^{2 n-2}(x, t) u_{x}^{2}(x, t) d x \leqq C, \quad 0 \leqq t \leqq T
$$

Proof. Using (3.3) and (3.18), noting that $\left[u_{x} / u\right]_{t}=\left[u_{t} / u\right]_{x}$, we may write (3.4) as follows:

$$
\left\{\beta r^{n-1} \frac{u_{x}}{u}-v\right\}_{t}=R r^{n-1}\left[\frac{\theta_{x}}{u}-\frac{\theta u_{x}}{u^{2}}\right]+\beta(n-1) r^{n-2} v \frac{u_{x}}{u} .
$$

Multiplying this equation by $\beta r^{n-1} u_{x} / u-v$ and integrate over $[0, k] \times[0, t]$, we deduce by (3.9)-(3.10), (3.28), Cauchy-Schwarz's inequality, and (3.19) that

$$
\begin{aligned}
& \int_{0}^{k}\left[\beta r^{n-1} \frac{u_{x}}{u}-v\right]^{2}(x, t) d x \leqq C+C \int_{0}^{t} \int_{0}^{k}\left\{r^{2 n-2} \frac{\left|\theta_{x} u_{x}\right|}{u^{2}}+r^{2 n-2} \theta \frac{u_{x}^{2}}{u^{3}}\right. \\
& \left.+r^{n-1} \frac{\left|\theta_{x} v\right|}{u}+r^{n-1} \theta \frac{\left|u_{x} v\right|}{u^{2}}+r^{2 n-3} v \frac{u_{x}^{2}}{u^{2}}+r^{n-2} v^{2}\left|\frac{u_{x}}{u}\right|\right\} d x d s \\
& \leqq C+C \int_{0}^{t}\left(1+\max _{[0, k]}\left(\theta^{2}+v^{2}\right)\right) \int_{0}^{k} r^{2 n-2} \frac{u_{x}^{2}}{u^{2}} d x d s+C \int_{0}^{t} \int_{0}^{k}\left(v^{2}+r^{2 n-2} \theta_{x}^{2}\right) d x d s,
\end{aligned}
$$

which together with (3.20) and (3.47) yields

$$
\begin{aligned}
& \int_{0}^{k}\left(r^{2 n-2} \frac{u_{x}^{2}}{u^{2}}\right)(x, t) d x \\
& \leqq C+C \int_{0}^{t}\left(1+\max _{[0, k]}\left(\theta^{2}+v^{2}\right)\right) \int_{0}^{k} r^{2 n-2} \frac{u_{x}^{2}}{u^{2}} d x d s \\
& \quad+C \int_{0}^{k} v^{2}(x, t) d x+C \int_{0}^{t} \int_{0}^{k}\left(v^{2}+r^{2 n-2} \theta_{x}^{2}\right) d x d s \\
& \leqq C+C \int_{0}^{t}\left(1+\max _{[0, k]}\left(\theta^{2}+v^{2}\right)\right) \int_{0}^{k} r^{2 n-2} \frac{u_{x}^{2}}{u^{2}} d x d s, \quad t \in[0, T] .
\end{aligned}
$$

Applying Gronwall's inequality to (3.53), utilizing (3.47), (3.51) and (3.28), we obtain (3.52). The proof is complete.

In the following lemma we estimate $v_{t}$ in the $L^{2}\left(Q_{T}^{k}\right)$-norm. The crucial step in the proof is to bound $\max _{[0, k]}\left|\left(r^{n-1} v\right)_{x} / u\right|$ by the $L^{2}\left(Q_{T}^{k}\right)$-norm of $\left(r^{n-1} v\right)_{x}$ and $v_{t}$, using Eq. (3.4) and Sobolev's imbedding theorem. 


\section{Lemma 3.10.}

$$
\int_{0}^{t} \int_{0}^{k} v_{t}^{2} d x d s+\int_{0}^{k}\left(r^{2 n-2} v_{x}^{2}+u_{t}^{2}\right)(x, t) d x \leqq C, \quad t \in[0, T] .
$$

Proof. Multiply (3.4) by $v_{t}$ and integrate over $[0, k] \times[0, t]$. After integrating by parts, and making use of (3.18), (3.28), and (3.47), (3.52), (3.9)-(3.10), and Eq. (3.3), we obtain

$$
\begin{aligned}
& \int_{0}^{t} \int_{0}^{k} v_{t}^{2} d x d s=-\beta \int_{0}^{t} \int_{0}^{k} \frac{\left(r^{n-1} v\right)_{x}}{u}\left[r^{n-1} v_{t}\right]_{x} d x d s-R \int_{0}^{t} \int_{0}^{k}\left[\frac{\theta}{u}\right]_{x} r^{n-1} v_{t} d x d s \\
& \leqq-\beta \int_{0}^{t} \int_{0}^{k} \frac{\left(r^{n-1} v\right)_{x}}{u}\left(r^{n-1} v\right)_{x t} d x d s+(n-1) \beta \int_{0}^{t} \int_{0}^{k} \frac{\left(r^{n-1} v\right)_{x}}{u}\left(r^{n-2} v^{2}\right)_{x} d x d s \\
& \quad+C \int_{0}^{t} \int_{0}^{k}\left\{r^{2 n-2} \theta_{x}^{2}+r^{2 n-2} \theta^{2} u_{x}^{2}\right\} d x d s+\frac{1}{2} \int_{0}^{t} \int_{0}^{k} v_{t}^{2} d x d s \\
& \leqq C-\frac{\beta}{2} \int_{0}^{k} \frac{\left(r^{n-1} v\right)_{x}^{2}}{u} d x+\int_{0}^{t} \int_{0}^{k}\left\{C \frac{\left|\left(r^{n-1} v\right)_{x}^{3}\right|}{u^{2}}+C \frac{\left|\left(r^{n-1} v\right)_{x}\left(r^{n-2} v^{2}\right)_{x}\right|}{u}+\frac{v_{t}^{2}}{2}\right\} d x d s .
\end{aligned}
$$

With the help of (3.28), (3.18)-(3.20), (3.55) implies

$$
\begin{aligned}
& \int_{0}^{t} \int_{0}^{k} v_{t}^{2} d x d s+\int_{0}^{k}\left(r^{n-1} v\right)_{x}^{2}(x, t) d x \\
& \quad \leqq C+C \int_{0}^{t} \max _{[0, k]} \frac{\left|\left(r^{n-1} v\right)_{x}\right|}{u} \int_{0}^{k}\left\{\left(r^{n-1} v\right)_{x}^{2}+v^{2}+|v| r^{n-2}\left|v_{x}\right|\right\} d x d s \\
& \quad \leqq C+C \int_{0}^{t} \max _{[0, k]} \frac{\left|\left(r^{n-1} v\right)_{x}\right|}{u} \int_{0}^{k}\left\{\left(r^{n-1} v\right)_{x}^{2}+v^{2}+|v|\left|\left(r^{n-1} v\right)_{x}\right|\right\} d x d s \\
& \quad \leqq C+C \int_{0}^{t} \max _{[0, k]} \frac{\left|\left(r^{n-1} v\right)_{x}\right|}{u}\left\{1+\int_{0}^{k}\left(r^{n-1} v\right)_{x}^{2} d x\right\} d s .
\end{aligned}
$$

From Sobolev's imbedding theorem $\left(H^{1}(0,1) \hookrightarrow L^{\infty}(0,1)\right.$, or $\left.W^{1,1}(0,1) \hookrightarrow L^{\infty}(0,1)\right)$, we get

$$
\begin{gathered}
\|\cdot\|_{L^{\infty}(i, i+1)} \leqq C\|\cdot\|_{H^{1}(i, i+1)} \leqq C\|\cdot\|_{H^{1}(0, k)} \quad \forall 0 \leqq i \leqq k-1, \text { or } \\
\|\cdot\|_{L^{\infty}(i, i+1)} \leqq C\|\cdot\|_{W^{1,1}(i, i+1)} \leqq C\|\cdot\|_{W^{1,1}(0, k)} \quad \forall 0 \leqq i \leqq k-1,
\end{gathered}
$$

with some constant $C$ being independent of $i$ and $k$. So the term $\max _{[0, k]}\left|\left(r^{n-1} v\right)_{x}\right| / u$ on the right-hand side of (3.56) can be estimated as follows, using (3.40), (3.28), 
(3.4) and (3.19),

$$
\begin{aligned}
& \max _{[i, i+1]} \frac{\left|\left(r^{n-1} v\right)_{x}(\cdot, t)\right|}{u} \leqq C \max _{[i, i+1]}|\sigma(\cdot, t)|+C \max _{[0, k]}\left(\frac{\theta}{u}\right)(\cdot, t) \\
& \leqq C\left(\|\sigma(t)\|_{L^{2}(i, i+1)}+\left\|\sigma_{x}(t)\right\|_{L^{2}(i, i+1)}\right)+C \max _{[i, i+1]} \theta(\cdot, t) \\
& \quad \leqq C\left(\left\|\left(r^{n-1} v\right)_{x}(t)\right\|_{L^{2}(i, i+1)}+\left\|r^{-n+1} v_{t}(t)\right\|_{L^{2}(i, i+1)}\right)+C \max _{[i, i+1]} \theta(\cdot, t) \\
& \leqq C\left(\left\|\left(r^{n-1} v\right)_{x}(t)\right\|_{L^{2}(0, k)}+\left\|v_{t}(t)\right\|_{L^{2}(0, k)}\right)+C \max _{[i, i+1]} \theta(\cdot, t), \quad 0 \leqq i \leqq k-1 .
\end{aligned}
$$

Inserting (3.58) into (3.56) and applying (3.47), we conclude

$$
\begin{gathered}
\int_{0}^{t} \int_{0}^{k} v_{t}^{2} d x d s+\int_{0}^{k}\left(r^{n-1} v\right)_{x}^{2}(x, t) d x \leqq C+\frac{1}{2} \int_{0}^{t} \int_{0}^{k} v_{t}^{2} d x d s \\
+C \int_{0}^{t}\left(\int_{0}^{k}\left(r^{n-1} v\right)_{x}^{2}(x, s) d x+\max _{[0, k]} \theta^{2}(\cdot, s)+1\right) \\
\quad \times \int_{0}^{k}\left(r^{n-1} v\right)_{x}^{2}(x, s) d x d s, \quad t \in[0, T]
\end{gathered}
$$

which together with Gronwall's inequality, (3.47) and (3.50) gives us

$$
\int_{0}^{t} \int_{0}^{k} v_{t}^{2} d x d s+\int_{0}^{k}\left(r^{n-1} v\right)_{x}^{2}(x, t) d x \leqq C \quad \forall t \in[0, T]
$$

Noting that by virtue of (3.18) and (3.28), $\left(r^{n-1} v\right)_{x}^{2} \geqq \frac{1}{2} r^{2 n-2} v_{x}^{2}-C v^{2}$, thus (3.59) combined with (3.20) and Eq. (3.3) yields (3.54). This completes the proof.

An immediate consequence of Lemma 3.10 is

$$
|v(x, t)| \leqq C, \quad 0 \leqq x \leqq k, 0 \leqq t \leqq T .
$$

In fact, (3.60) easily follows from $(3.57)_{1},(3.19)-(3.20)$ and (3.54).

Another corollary of Lemma 3.10 is the following.

\section{Lemma 3.11.}

$$
\int_{0}^{T} \int_{0}^{k} r^{4 n-4} v_{x x}^{2} d x d s+\int_{0}^{T} \int_{0}^{k} r^{2 n-2} u_{x t}^{2} d x d s+\int_{0}^{T} \max _{[0, k]}\left(r^{n-1} v\right)_{x}^{2}(\cdot, s) d s \leqq C .
$$

Proof. From Eq. (3.4) we get

$$
\beta \frac{r^{n-1}\left(r^{n-1} v\right)_{x x}}{u}=v_{t}+\beta \frac{r^{n-1}\left(r^{n-1} v\right)_{x} u_{x}}{u^{2}}+R r^{n-1}\left(\frac{\theta}{u}\right)_{x}
$$


So if we use (3.28), Lemma 3.10, (3.52), and (3.57) $2,(3.47),(3.3)$, and (3.19), we obtain

$$
\begin{aligned}
\int_{0}^{T} \int_{0}^{k} r^{2 n-2}\left(r^{n-1} v\right)_{x x}^{2} d x d s \leqq & C+C \int_{0}^{T} \max \left|\left(r^{n-1} v\right)_{x}\right|^{2} d s \\
& +C \int_{0}^{T} \int_{0}^{k} r^{2 n-2}\left[\theta_{x}^{2}+u_{x}^{2} \theta^{2}\right] d x d s \\
\leqq & C+C \int_{0}^{T} \int_{0}^{k}\left\{\left(r^{n-1} v\right)_{x}^{2}+\left|\left(r^{n-1} v\right)_{x}\right|\left|\left(r^{n-1} v\right)_{x x}\right|\right\} d x d s \\
\leqq & C+C \int_{0}^{T} \int_{0}^{k} u_{t}^{2} d x d s+\frac{a^{2 n-2}}{2} \int_{0}^{T} \int_{0}^{k}\left(r^{n-1} v\right)_{x x}^{2} d x d s \\
\leqq & C+\frac{1}{2} \int_{0}^{T} \int_{0}^{k} r^{2 n-2}\left(r^{2 n-2} v\right)_{x x}^{2} d x d s,
\end{aligned}
$$

whence

$$
\int_{0}^{T} \int_{0}^{k} r^{2 n-2}\left(r^{n-1} v\right)_{x x}^{2} d x d s+\int_{0}^{T} \int_{0}^{k} r^{2 n-2} u_{x t}^{2} d x d s \leqq C,
$$

where we have also used Eq. (3.3). In view of (3.18)-(3.19), (3.28), (3.60), and $(3.57)_{1}$, we see that

$$
\begin{aligned}
\frac{1}{2} r^{4 n-4} v_{x x}^{2} \leqq & r^{2 n-2}\left(r^{n-1} v\right)_{x x}^{2}+C\left(v^{2}+r^{2 n-2} u_{x}^{2}+r^{2 n-2} v_{x}^{2}\right) \\
\max _{[0, k]}\left|\left(r^{n-1} v\right)_{x}(\cdot, t)\right|^{2} \leqq & C\left(\left\|\left(r^{n-1} v\right)_{x}(t)\right\|_{L^{2}(0, k)}^{2}+\left\|\left(r^{n-1} v\right)_{x x}(t)\right\|_{L^{2}(0, k)}^{2}\right) \\
\leqq & \|v(t)\|_{L^{2}(0, k)}+\left\|r^{n-1} v_{x}(t)\right\|_{L^{2}(0, k)}^{2} \\
& +\left\|r^{n-1}\left(r^{n-1} v\right)_{x x}(t)\right\|_{L^{2}(0, k)}^{2}, \quad t \in[0, T]
\end{aligned}
$$

Combining (3.62) and (3.63), and utilizing (3.20), (3.52) and (3.48), we obtain (3.61). The proof of the lemma is complete.

Similarly to Lemma 3.10 we can show the following estimates for $\theta$ :

Lemma 3.12. We have

$$
\begin{gathered}
\int_{0}^{t} \int_{0}^{k} \theta_{t}^{2} d x d s+\int_{0}^{k} r^{2 n-2} \theta_{x}^{2}(x, t) d x \leqq C, \quad 0 \leqq t \leqq T ; \\
\underline{\theta} \leqq \theta(x, t) \leqq \bar{\theta} \quad \text { for any } x \in[0, k], t \in[0, T],
\end{gathered}
$$

where $\underline{\theta}$ and $\bar{\theta}$ are positive constants independent of $k$.

Proof. Multiply (3.5) by $\theta_{t}$ and integrate over $[0, k] \times[0, t](0 \leqq t \leqq T)$. Integrating by parts, applying Cauchy-Schwarz's inequality, and using (3.10), 
(3.18)-(3.19), (3.28), (3.60), and (3.47), (3.54), (3.20), (3.61), and Eq. (3.3) we get

$$
\begin{aligned}
& \frac{c_{V}}{2} \int_{0}^{t} \int_{0}^{k} \theta_{t}^{2} d x d s+\frac{\kappa_{Q}}{2} \int_{0}^{k} \frac{r^{2 n-2} \theta_{x}^{2}(x, t)}{u(x, t)} d x \\
& \leqq C+C \int_{0}^{t} \int_{0}^{k}\left\{r^{2 n-2} \theta_{x}^{2}\left|u_{t}\right|+\left(r^{n-1} v\right)_{x}^{4}+\theta^{2}\left(r^{n-1} v\right)_{x}^{2}+\left(r^{n-2} v^{2}\right)_{x}^{2}\right\} d x d s \\
& \leqq C+C \int_{0}^{t} \max _{[0, k]} u_{t}^{2} \int_{0}^{k} r^{2 n-2} \theta_{x}^{2} d x d s \\
& \quad+C \int_{0}^{t} \max _{[0, k]}\left\{\left(r^{n-1} v\right)_{x}^{2}+\theta^{2}+1\right\} \int_{0}^{k}\left(r^{2 n-2} v_{x}^{2}+v^{2}\right) d x d s \\
& \leqq C+C \int_{0}^{t} \max _{[0, k]}\left(r^{n-1} v\right)_{x}^{2} \int_{0}^{k} \frac{r^{2 n-2} \theta_{x}^{2}}{u} d x d s, \quad t \in[0, T]
\end{aligned}
$$

Applying Gronwall's inequality to (3.66), and recalling (3.61) and (3.28), we obtain (3.64).

To show (3.65) we make use of $(3.57)_{1},(3.19),(3.47)$ and (3.64) to deduce

$$
\begin{aligned}
\theta^{2}(x, t) & \leqq 2(\theta(x, t)-1)^{2}+2 \leqq 2+C \int_{0}^{k}\left((\theta-1)^{2}+\theta_{x}^{2}\right)(x, t) d x \\
& \leqq 2+C \int_{0}^{k}\left((\theta-1)^{2}+r^{2 n-2} \theta_{x}^{2}\right)(x, t) d x \leqq C, \quad x \in[0, k], t \in[0, T] .
\end{aligned}
$$

To get a lower bound of $\theta(x, t)$ we adapt and modify an idea of Alikakos [2] for parabolic equations. Multiplying (3.5) by $-1 / \theta^{2}$, using (3.23) and taking

$$
-\kappa_{Q}\left[\frac{r^{2 n-2} \theta_{x}}{u}\right]_{x} \frac{1}{\theta^{2}}=\kappa_{Q}\left[\frac{r^{2 n-2}}{u}\left(\frac{1}{\theta}\right)_{x}\right]_{x}-2 \kappa_{Q} \frac{r^{2 n-2} \theta_{x}^{2}}{u \theta^{3}}
$$

into account, we see that

$$
c_{V}\left[\frac{1}{\theta}\right]_{t} \leqq \kappa_{Q}\left[\frac{r^{2 n-2}}{u}\left(\frac{1}{\theta}\right)_{x}\right]_{x}+R \frac{\left(r^{n-1} v\right)_{x}}{u \theta}, \quad x \in[0, k], t \in[0, T] .
$$

If we multiply (3.68) by $j \theta^{-j+1}(j \geqq 2$ integer), integrate over $[0, k] \times[0, t]$, employ a partial integration with respect to $x$, and use (3.28), we find

$$
\left\|\frac{1}{\theta(t)}\right\|_{L^{j}(0, k)}^{j} \leqq\left\|\frac{1}{\theta^{0}}\right\|_{L^{j}(0, k)}^{j}+C j \int_{0}^{t} \max _{[0, k]}\left|\left(r^{n-1} v\right)_{x}\right|\left\|\frac{1}{\theta(s)}\right\|_{L^{j}(0, k)}^{j} d s,
$$

where the constant $C$ is independent of $j$ and $k$. An application of Gronwall's inequality to (3.69) and use of (3.61) yields

$$
\left\|\frac{1}{\theta(t)}\right\|_{L^{\prime}(0, k)}^{j} \leqq\left\|\frac{1}{\theta^{0}}\right\|_{L^{j}(0, k)}^{j} e^{C(1+T) j} \quad \forall t \in[0, T],
$$


which, by taking the $1 / j^{\text {th }}$ power and then passing to the limit as $j \rightarrow \infty$, implies $\|1 / \theta(t)\|_{L^{\infty}(0, k)} \leqq C\left\|1 / \theta^{0}\right\|_{L^{\infty}} \leqq C$ for any $t \in[0, T]$. This together with (3.67) gives (3.65).

As a result of Lemma 3.12 one has

\section{Lemma 3.13.}

$$
\int_{0}^{T} \int_{0}^{k} r^{4 n-4} \theta_{x x}^{2} d x d s+\int_{0}^{T} \max _{[0, k]}\left[r^{2 n-2} \theta_{x}^{2}\right](\cdot, s) d s \leqq C .
$$

Proof. From Eq. (3.5), (3.18)-(3.19), (3.28), (3.47), and (3.52), (3.60), (3.20), (3.54), and (3.61), we get (cf. the proof of (3.66))

$$
\begin{aligned}
& \int_{0}^{T} \int_{0}^{k} r^{4 n-4} \theta_{x x}^{2} d x d s \\
& \quad \leqq C \int_{0}^{T} \int_{0}^{k}\left\{\theta_{t}^{2}+r^{2 n-2} \theta_{x}^{2}+r^{4 n-4} \theta_{x}^{2} u_{x}^{2}+\left(r^{n-1} v\right)_{x}^{4}+\theta^{2}\left(r^{n-1} v\right)_{x}^{2}+\left(r^{n-2} v^{2}\right)_{x}^{2}\right\} d x d s \\
& \quad \leqq C+C \int_{0}^{T} \max _{[0, k]} r^{2 n-2} \theta_{x}^{2}(\cdot, s) d s .
\end{aligned}
$$

Here the second term on the right-hand side of (3.71) can be estimated as follows, using $(3.57)_{2}$ and (3.47),

$$
\begin{aligned}
C \int_{0}^{T} \max _{[0, k]} r^{2 n-2} \theta_{x}^{2} d s & \leqq C \int_{0}^{T} \int_{0}^{k}\left\{r^{2 n-2} \theta_{x}^{2}+\left|r^{n-1} \theta_{x}\right|\left|\left[r^{n-1} \theta_{x}\right]_{x}\right|\right\} d x d s \\
& \leqq C \int_{0}^{T} \int_{0}^{k} r^{2 n-2} \theta_{x}^{2} d x d s+\frac{a^{2 n-2}}{2} \int_{0}^{T} \int_{0}^{k} r^{2 n-2} \theta_{x x}^{2} d x d s \\
& \leqq C+\frac{1}{2} \int_{0}^{T} \int_{0}^{k} r^{4 n-4} \theta_{x x}^{2} d x d s
\end{aligned}
$$

Substituting (3.72) into (3.71), we get (3.70). The proof is complete.

From (3.28), Taylor's theorem and (3.20), it follows that $\|u(t)-1\|_{L^{2}(0, k)} \leqq C$ for all $t \in[0, T]$. This, together with Lemmas 3.4, 3.6-3.13, yields (3.16) in Theorem 3.3.

Now we turn to the case that $u_{0}, v_{0}, \theta_{0}$ satisfy (2.18). In this case we proceed to get estimates of high derivatives of $u, v, \theta$ in the $L^{2}$-norm.

Using (3.18), we differentiate (3.4) with respect to $t$, multiply the resulting equation by $v_{t}$ and then integrate. We integrate by parts with respect to $x$ to arrive at

$$
\begin{gathered}
\frac{1}{2} \int_{0}^{k} v_{t}^{2}(x, t) d x=\frac{1}{2} \int_{0}^{k} v_{t}^{2}(x, 0) d x-\int_{0}^{t} \int_{0}^{k}\left[\frac{\beta}{u}\left(r^{n-1} v\right)_{x}-R \frac{\theta}{u}\right]_{t}\left[r^{n-1} v_{t}\right]_{x} d x d s \\
-(n-1) \int_{0}^{t} \int_{0}^{k}\left[\frac{\beta}{u}\left(r^{n-1} v\right)_{x}-R \frac{\theta}{u}\right]\left[r^{n-2} v v_{t}\right]_{x} d x d s, \quad t \in[0, T] .
\end{gathered}
$$


It should be pointed out here that the derivation of (3.73) is informal because of the lack of regularity in some steps. However, the rigorous derivation can be achieved by using difference quotient (with respect to $t$ ) and taking to the limit (cf. Renardy, Hrusa and Nohel [24, pp. 145-163]), or by using mollifiers.

If we apply Lemma 3.1, (3.18)-(3.19), (3.28), (3.60), and (3.65), CauchySchwarz's inequality, (3.20), (3.54), (3.61), and (3.64) to (3.73), we infer

$$
\begin{aligned}
& \int_{0}^{k} v_{t}^{2}(x, t) d x+\int_{0}^{t} \int_{0}^{k} r^{2 n-2} v_{x t}^{2} d x d s \\
& \leqq C+C \int_{0}^{t} \int_{0}^{k}\left\{v^{2}+v_{t}^{2}+r^{2 n-2} v_{x}^{2}+u_{t}^{2}+\left(r^{n-1} v\right)_{x}^{2} r^{2 n-2} v_{x}^{2}+\theta_{t}^{2}\right\} \\
& \leqq C, \quad t \in[0, T] .
\end{aligned}
$$

Similarly, differentiating (3.3) with respect to $t$ we obtain

$$
\int_{0}^{T} \int_{0}^{k} u_{t t}^{2} d x d s \leqq C \int_{0}^{T} \int_{0}^{k}\left\{v^{2}+v_{t}^{2}+r^{2 n-2} v_{x}^{2}+u_{t}^{2}+r^{2 n-2} v_{x t}^{2}\right\} d x d s \leqq C .
$$

It follows from (3.4), (3.65), (3.74), (3.52), and (3.64), (3.57) 2 , and (3.59) that

$$
\begin{aligned}
& \int_{0}^{k} r^{2 n-2}\left(r^{n-1} v\right)_{x x}^{2}(x, t) d x \\
& \quad \leqq C \int_{0}^{k}\left\{v_{t}^{2}+r^{2 n-2} u_{x}^{2}\left[\left(r^{n-1} v\right)_{x}^{2}+1\right]+r^{2 n-2} \theta_{x}^{2}\right\}(x, t) d x \\
& \quad \leqq C+C \max _{[0, k]}\left(r^{n-1} v\right)_{x}^{2}(\cdot, t) \leqq C+C \int_{0}^{k}\left\{\left(r^{n-1} v\right)_{x}^{2}+\left|\left(r^{n-1} v\right)_{x}\right|\left|\left(r^{n-1} v\right)_{x x}\right|\right\} d x \\
& \quad \leqq C+\frac{1}{2} \int_{0}^{k} r^{2 n-2}\left(r^{n-1} v\right)_{x x}^{2}(x, t) d x, \quad t \in[0, T],
\end{aligned}
$$

which combined with $(3.63)_{1},(3.20),(3.52)$ and (3.54) implies

$$
\int_{0}^{k} r^{4 n-4}(x, t) v_{x x}^{2}(x, t) d x \leqq C \text { for all } t \in[0, T] .
$$

From this and (3.74) we conclude:

\section{Lemma 3.14.}

$$
\int_{0}^{k} v_{t}^{2}(x, t) d x+\int_{0}^{k} r^{4 n-4} v_{x x}^{2}(x, t) d x+\int_{0}^{t} \int_{0}^{k} r^{2 n-2} v_{x t}^{2} d x d s \leqq C \quad \forall t \in[0, T] .
$$

It is easy to see that by $(3.57)_{1},(3.19),(3.3),(3.59)$ and (3.76),

$$
\begin{aligned}
& \left(r^{n-1} v\right)_{x}^{2}(x, t)+\int_{0}^{k} r^{2 n-2} u_{x t}^{2}(x, t) d x \\
& \quad \leqq C \int_{0}^{k}\left\{\left(r^{n-1} v\right)_{x}^{2}+r^{2 n-2}\left(r^{n-1} v\right)_{x x}^{2}\right\}(x, t) d x \leqq C
\end{aligned}
$$

for any $x \in[0, k]$ and $t \in[0, T]$. 
We differentiate Eq. (3.5) with respect to $t$, multiply by $\theta_{t}$ and integrate over $[0, k] \times[0, t](0 \leqq t \leqq T)$. Using (3.77)-(3.78), following the same procedure as used in Lemma 3.14 we obtain the following lemma the proof of which will be omitted here.

\section{Lemma 3.15.}

$$
\int_{0}^{k} \theta_{t}^{2}(x, t) d x+\int_{0}^{k} r^{4 n-4} \theta_{x x}^{2}(x, t) d x+\int_{0}^{t} \int_{0}^{k} r^{2 n-2} \theta_{x t}^{2} d x d s \leqq C \quad \forall t \in[0, T] .
$$

From Lemmas 3.14-3.15, (3.75) and (3.78) we get (3.17) immediately. The proof of Theroem 3.3 is complete.

\section{Proof of Theorem 2.1}

In this section let $\left\{u_{k}, v_{k}, \theta_{k}\right\}$ denote the solution of (3.3)-(3.8). We first prove the existence.

I. Existence. Let $\phi_{k}$ be the same as in Sect. 3. For $k \in \mathbb{N}$ we define

$$
\begin{aligned}
& u_{k}(x, t):=\left(u_{k}(x, t)-1\right) \phi_{k}(x)+1, \quad \tilde{v}_{k}(x, t):=v_{k}(x, t) \phi_{k}(x), \\
& \tilde{\theta}_{k}(x, t):=\left(\theta_{k}(x, t)-1\right) \phi_{k}(x)+1, \quad x \in[0, \infty), t \in[0, T] .
\end{aligned}
$$

Then for any $l \in \mathbb{N}$ we have

$$
\begin{aligned}
\left\{u_{k}(x, t), \tilde{v}_{k}(x, t), \tilde{\theta}_{k}(x, t)\right\}= & \left\{u_{k}(x, t), v_{k}(x, t), t_{k}(x, t)\right\} \\
& \text { for all }(x, t) \in[0, l] \times[0, T] \text { and } k \geqq 2 l .
\end{aligned}
$$

By virtue of (3.16) in Theorem 3.3 we have

$$
\begin{aligned}
& \left\|\left(u_{k}-1, \tilde{v}_{k}, \tilde{\theta}_{k}-1\right)\right\|_{L^{\infty}\left([0, T], H^{1}\right)}+\left\|\partial_{t} u_{k}\right\|_{L^{\infty}\left([0, T], L^{2}\right)}+\left\|\left(\tilde{v}_{k}, \tilde{\theta}_{k}-1\right)\right\|_{L^{2}\left([0, T], H^{2}\right)} \\
& \quad+\left\|\left(\partial_{t} u_{k}, \partial_{t} \tilde{v}_{k}, \partial_{t} \tilde{\theta}_{k}, \partial_{t} \partial_{x} u_{k}\right)\right\|_{L^{2}\left(Q_{T}\right)} \leqq C \quad \text { for any } k \geqq 1
\end{aligned}
$$

In view of (4.3) there are functions $u, v, \theta$, and a subsequence of $\left\{u_{k}, \tilde{v}_{k}, \tilde{\theta}_{k}\right\}$, still denoted by $\left\{u_{k}, \tilde{v}_{k}, \tilde{\theta}_{k}\right\}$, such that as $k \rightarrow \infty$,

$$
\begin{aligned}
& \left(u_{k}-1, \tilde{v}_{k}, \tilde{\theta}_{k}-1,\left(u_{k}\right)_{t},\left(u_{k}\right)_{x},\left(\tilde{v}_{k}\right)_{x},\left(\tilde{\theta}_{k}\right)_{x}\right) \\
& \rightarrow\left(u-1, v, \theta-1, u_{t}, u_{x}, v_{x}, \theta_{x}\right) \quad(\text { weak-* }) \text { in } L^{\infty}\left([0, T], L^{2}\right), \\
& \left(\left(u_{k}\right)_{t},\left(\tilde{v}_{k}\right)_{t},\left(\tilde{\theta}_{k}\right)_{t},\left(u_{k}\right)_{x t}\right) \rightarrow\left(u_{t}, v_{t}, \theta_{t}, u_{x t}\right) \quad(\text { weakly }) \text { in } L^{2}\left(Q_{T}\right), \\
& \left(\tilde{v}_{k}, \tilde{\theta}_{k}-1\right) \rightarrow(v, \theta-1) \quad(\text { weakly }) \text { in } L^{2}\left([0, T], H^{2}\right) ;
\end{aligned}
$$

and

$$
\|(u-1, v, \theta-1)\|_{L^{\infty}\left([0, T], H^{1}\right)}+\left\|u_{t}\right\|_{L^{\infty}\left([0, T], L^{2}\right)}+\left\|\left(v_{t}, \theta_{t}, u_{x t}, v_{x x}, \theta_{x x}\right)\right\|_{L^{2}\left(Q_{T}\right)} \leqq C .
$$

Furthermore by Rellich's selection theorem and the diagonal procedure, we may extract a subsequence of $\left\{u_{k}, \tilde{v}_{k}, \tilde{\theta}_{k}\right\}$, still denoted by $\left\{u_{k}, \tilde{v}_{k}, \tilde{\theta}_{k}\right\}$, such that for any 
$l \in \mathbb{N}$, we have

$$
\left\{u_{k}, \tilde{v}_{k}, \tilde{\theta}_{k}\right\} \rightarrow\{u, v, \theta\} \quad \text { strongly in } L^{2}((0, l) \times(0, T)) .
$$

Define

$$
\begin{gathered}
r(x, t):=r_{0}(x)+\int_{0}^{t} v(x, \tau) d \tau, \\
r_{0}(x):=\left\{a^{n}+n \int_{0}^{x} u_{0}(y) d y\right\}^{1 / n}, \quad x, t \geqq 0 .
\end{gathered}
$$

Next we prove that $\{u, v, \theta\}$ obtained in (4.4), together with $r(x, t)$ defined by (4.7), is a generalized solution of (2.10)-(2.15).

The first observation is that by virtue of (4.5) and Sobolev's imbedding theorem, $u-1, v, \theta-1 \in C^{0}\left([0, T], L^{2}\right) \cap L^{\infty}\left([0, T], H^{1}\right)$. From this and the interpolation inequality $\left(\|\cdot\|_{H^{\gamma}} \leqq C\|\cdot\|^{1-\gamma}\|\cdot\|_{H^{\gamma}}^{\gamma}, 0 \leqq \gamma<1\right)$, it follows that $u-1, v$, $\theta-1 \in C^{0}\left([0, T], H^{\gamma}\right)$ for any $\gamma \in[0,1)$. So, with the help of Sobolev's imbedding theorem $\left(H^{\gamma} \hookrightarrow C^{0}\right.$ for $\left.\gamma>1 / 2\right)$, one finds

$$
u, v, \theta \in C^{0}([0, \infty) \times[0, T]) .
$$

By (3.28), (3.65), (4.1), (4.6) and (4.8) we conclude

$$
\underline{u} \leqq u(x, t) \leqq \bar{u}, \quad \underline{\theta} \leqq \theta(x, t) \leqq \bar{\theta} \quad \forall(x, t) \in[0, \infty) \times[0, T] .
$$

Let $\varphi \in C_{0}^{\infty}(0, \infty)$ be arbitrary but fixed, and let $\operatorname{supp} \varphi \subset[0, L]$ for some $L$. Then from (4.2), (4.4) 1 and (3.10) we see that for any $k \geqq 2 L$,

$$
\begin{aligned}
\left(u(0)-u_{0}, \varphi\right)= & \left(u(0)-u_{k}(0), \varphi\right)+\left(u_{k}(0)-u_{0}, \varphi\right) \\
= & \frac{1}{T} \int_{0}^{T}\left(\partial_{t} u(t)-\partial_{t} u_{k}(t), \varphi\right)(t-T) d t \\
& +\frac{1}{T} \int_{0}^{T}\left(u(t)-u_{k}(t), \varphi\right) d t+\left(u_{k}^{0}-u_{0}, \varphi\right) \rightarrow 0 \quad \text { as } k \rightarrow \infty,
\end{aligned}
$$

which yields $\left.u\right|_{t=0}=u_{0}$. In the same manner we can show $\left.(v, \theta)\right|_{t=0}=\left(v_{0}, \theta_{0}\right)$. Therefore, $\{u, v, \theta\}$ satisfies the initial condition (2.13). To show that $v, \theta$ also satisfy the boundary conditions (2.14), we note that by virtue of $(4.5)$ and $(4.8), v(0, \cdot) \in$ $C^{0}([0, T])$ and $\theta_{x}(0, \cdot) \in L^{2}(0, T)$ are well-defined for $t \in[0, T]$. On account of (3.7), (4.2) and (4.4) we conclude that for any $\eta \in C_{0}^{\infty}(0, T)$,

$$
\begin{aligned}
\int_{0}^{T} v(0, t) \eta(t) d t= & \int_{0}^{T}\left(v(0, t)-\tilde{v}_{k}(0, t)\right) \eta(t) d t \\
= & \int_{0}^{T} \int_{0}^{1}\left(v-\tilde{v}_{k}\right)_{x}(x, t)(x-1) \eta(t) d x d t \\
& +\int_{0}^{T} \int_{0}^{1}\left(v-\tilde{v}_{k}\right)(x, t) \eta(t) d x d t \rightarrow 0 \quad \text { as } k \rightarrow \infty,
\end{aligned}
$$


which implies $v(0, t)=0$ for $t \in[0, T]$. In the same way one gets $\theta_{x}(0, t)=0$ for $t \in[0, T]$.

From (4.2), (3.10)-(3.12), (3.19), and (4.6)-(4.7), it follows that for any $l \in \mathbb{N}$,

$$
\begin{gathered}
r_{k} \rightarrow r \quad \text { strongly in } L^{2}((0, l) \times(0, T)) \text { as } k \rightarrow \infty, \\
a \leqq r(x, t) \text { for any }(x, t) \in[0, \infty) \times[0, T] .
\end{gathered}
$$

Let $\psi \in C_{0}^{\infty}\left(Q_{T}\right)$ be arbitrary but fixed, let $\operatorname{supp} \psi \subset[0, L] \times[0, T]$ for some $L$. Then by (4.2), (3.47), Cauchy-Schwarz's inequality, (4.10), and (4.4) we find that for any $k \geqq 2 L$,

$$
\begin{aligned}
\left\langle r^{n-1} \theta_{x}, \psi\right\rangle_{Q_{T}}= & \left\langle r^{n-1}\left(\theta-\tilde{\theta}_{k}\right)_{x}, \psi\right\rangle_{Q_{T}}+\left\langle\left(r^{n-1}-r_{k}^{n-1}\right)\left(\tilde{\theta}_{k}\right)_{x}, \psi\right\rangle_{Q_{T}}+\left\langle r_{k}^{n-1}\left(\theta_{k}\right)_{x}, \psi\right\rangle_{Q_{T}} \\
\leqq & \left\langle r^{n-1}\left(\theta-\tilde{\theta}_{k}\right)_{x}, \psi\right\rangle_{Q_{T}}+\left\langle\left(r^{n-1}-r_{k}^{n-1}\right)\left(\tilde{\theta}_{k}\right)_{x}, \psi\right\rangle_{Q_{T}}+C\|\psi\|_{L^{2}\left(Q_{T}\right)} \\
& \rightarrow C\|\psi\|_{L^{2}\left(Q_{T}\right)} \quad \text { as } k \rightarrow \infty
\end{aligned}
$$

which gives $r^{n-1} \theta_{x} \in L^{2}\left(Q_{T}\right)$. Analogously, we obtain $r^{2 n-2} \theta_{x x} \in L^{2}\left(Q_{T}\right)$. Therefore, to show that $\{u, v, \theta\}$ is a generalized solution it remains to show that $u, v, \theta$ satisfy Eqs. (2.10)-(2.12). To this end let $\psi \in C_{0}^{\infty}\left(Q_{T}\right)$ with $\operatorname{supp} \psi \subset$ $[0, L] \times[0, T]$ for some $L$, we have by (4.4), (4.2), (3.3), (4.6) and (4.10) that as $k \rightarrow \infty$,

$$
\begin{gathered}
\left\langle\partial_{t} u, \psi\right\rangle_{Q_{T}} \leftarrow\left\langle\partial_{t} \tilde{u}_{k}, \psi\right\rangle_{Q_{T}}=\left\langle\partial_{t} u_{k}, \psi\right\rangle_{Q_{T}}=\left\langle\left(r_{k}^{n-1} v_{k}\right)_{x}, \psi\right\rangle_{Q_{T}} \\
=-\left\langle r_{k}^{n-1} v_{k}, \psi_{x}\right\rangle_{Q_{T}} \rightarrow-\left\langle r^{n-1} v, \psi_{x}\right\rangle_{Q_{T}}=\left\langle\left(r^{n-1} v\right)_{x}, \psi\right\rangle_{Q_{T}}
\end{gathered}
$$

which gives

$$
u_{t}=\left(r^{n-1} v\right)_{x} \text { in } L^{2}\left(Q_{T}\right)
$$

with $r(x, t)$ being defined by (4.7). Recalling the definition (4.7) and using (4.13), we deduce by the same argument as used in the derivation of (3.18) that

$$
r^{n-1}(x, t) r_{x}(x, t)=u(x, t), \quad x \in[0, \infty), t \in[0, T] .
$$

In view of (4.2), (3.3), (4.4) and (4.13), we see that for any $l \in \mathbb{N}$,

$$
\begin{aligned}
& \left(r_{k}^{n-1} v_{k}\right)_{x}=\left(u_{k}\right)_{t}=\left(\tilde{u}_{k}\right)_{t} \rightarrow u_{t}=\left(r^{n-1} v\right)_{x} \\
& \text { weakly in } L^{2}((0, l) \times(0, T)) \text { as } k \rightarrow \infty .
\end{aligned}
$$

For any $\psi \in C_{0}^{\infty}\left(Q_{T}\right)$ with supp $\psi \subset[0, L] \times[0, T]$ for some $L$ we infer by (3.3), (4.2), integration by parts with respect to $x$, and (3.28), (4.9), (4.3)-(4.6), and 
(4.13), (4.15), (3.52), (3.59), (3.61) that for any $k \geqq 2 L$,

$$
\begin{aligned}
\left\langle\frac{\left(r_{k}^{n-1} v_{k}\right)_{x}^{2}}{u_{k}}, \psi\right\rangle_{Q_{T}} & =\left\langle\left(r_{k}^{n-1} v_{k}\right)_{x}^{2}\left(\frac{1}{u_{k}}-\frac{1}{u}\right), \psi\right\rangle_{Q_{T}}+\left\langle\frac{\left(\tilde{u}_{k}\right)_{t}\left(r_{k}^{n-1} \tilde{v}_{k}\right)_{x}}{u}, \psi\right\rangle_{Q_{T}} \\
= & \left\langle\left(r_{k}^{n-1} v_{k}\right)_{x}^{2}\left(\frac{1}{\tilde{u}_{k}}-\frac{1}{u}\right), \psi\right\rangle_{Q_{T}}-\left\langle\left(\tilde{u}_{k}\right)_{t x} r_{k}^{n-1} \tilde{v}_{k}, \frac{\psi}{u}\right\rangle_{Q_{T}} \\
& -\left\langle\left(\tilde{u}_{k}\right)_{t} r_{k}^{n-1} \tilde{v}_{k},\left(\frac{\psi}{u}\right)_{x}\right\rangle_{Q_{T}} \rightarrow-\left\langle u_{t x} r^{n-1} v, \frac{\psi}{u}\right\rangle_{Q_{T}} \\
& -\left\langle u_{t} r^{n-1} v,\left(\frac{\psi}{u}\right)_{x}\right\rangle_{Q_{T}}=\left\langle\frac{\left(r^{n-1} v\right)_{x}^{2}}{u}, \psi\right\rangle_{Q_{T}} .
\end{aligned}
$$

Similarly,

$$
\begin{aligned}
\left\langle\left(r_{k}^{n-2} v_{k}^{2}\right)_{x}, \psi\right\rangle_{Q_{T}} & \rightarrow\left\langle\left(r^{n-2} v^{2}\right)_{x}, \psi\right\rangle_{Q_{T}},\left\langle r_{k}^{n-1}\left[\frac{\left(r_{k}^{n-1} v_{k}\right)_{x}}{u_{k}}\right]_{x}, \psi\right\rangle_{Q_{T}} \\
& \rightarrow\left\langle r^{n-1}\left[\frac{\left(r^{n-1} v\right)_{x}}{u}\right]_{x}, \psi\right\rangle_{Q_{T}} .
\end{aligned}
$$

Now multiplying (3.3)-(3.5) by $\psi \in C_{0}^{\infty}\left(Q_{T}\right)$, passing to the limit as $k \rightarrow \infty$, applying (4.2), (4.4), (4.6), (4.15)-(4.17), and (4.13), we conclude that $\{u, v, \theta\}$ satisfies $(2.10)-(2.14)$. Thus we have proved the existence.

II. Uniqueness. Let $\{u, v, \theta\}$ and $\{\tilde{u}, \tilde{v}, \tilde{\theta}\}$ be generalized solutions of (2.10)-(2.15) satisfying (2.17) and $u, \theta, \tilde{u}, \tilde{\theta}>0$ on $\bar{Q}_{T}$. Then by Sobolev's imbedding theorem $\left(H^{1} \hookrightarrow L^{\infty}\right)$, we obtain

$$
\begin{array}{r}
C^{-1} \leqq u(x, t), \theta(x, t), \tilde{u}(x, t), \tilde{\theta}(x, t) \leqq C, \\
|v(x, t)|+|\tilde{v}(x, t)| \leqq C \quad \forall(x, t) \in[0, \infty) \times[0, T] .
\end{array}
$$

Here and throughout the proof of the uniqueness $C$ denotes a generic positive constant that may depend on $u, v, \theta, \tilde{u}, \tilde{v}$ and $\tilde{\theta}$.

Integration of (4.14) over [0,x] in the variable $x$ and use of (4.18) yield (note $r>0$, see the proof of (3.19))

$$
C^{-1}(1+x)^{1 / n} \leqq r(x, t) \leqq C(1+x)^{1 / n} \quad \forall(x, t) \in[0, \infty) \times[0, T]
$$

By (4.14), (4.19), (2.17) and Sobolev's imbedding theorem $\left(H^{1} \hookrightarrow L^{\infty}\right)$, we infer

$$
\int_{0}^{T} \max _{[0, \infty)}\left\{r^{2 n-2} \theta_{x}^{2}+\tilde{r}^{2 n-2} \tilde{\theta}_{x}^{2}+u_{t}^{2}+\tilde{u}_{t}^{2}\right\}(s) d s \leqq C .
$$

From (4.18) we see that $C^{-1} \tilde{u}(x, t) \leqq u(x, t) \leqq C \tilde{u}(x, t)$, which gives

$$
C^{-1} \tilde{r}^{n-1}(x, t) \tilde{r}_{x}(x, t) \leqq r^{n-1}(x, t) r_{x}(x, t) \leqq C \tilde{r}^{n-1}(x, t) \tilde{r}_{x}(x, t),
$$


where $\tilde{r}(x, t):=r_{0}(x)+\int_{0}^{t} \tilde{v}(x, \tau) d \tau$. Integrating the above inequality over $[0, x]$ and recalling $\tilde{r}(0, t)=r(0, t)=a$ we get

$$
C^{-1} \tilde{r}(x, t) \leqq r(x, t) \leqq C \tilde{r}(x, t) \quad \forall(x, t) \in[0, \infty) \times[0, T] .
$$

Let $\{U, V, \Theta\}:=\{u-\tilde{u}, v-\tilde{v}, \theta-\tilde{\theta}\}$. Then $U, V, \Theta$ satisfy

$$
\begin{aligned}
U_{t}= & \left(r^{n-1} V\right)_{x}+\left[\left(r^{n-1}-\tilde{r}^{n-1}\right) \tilde{v}\right]_{x} \\
V_{t}= & \beta r^{n-1}\left[\frac{\left(r^{n-1} V\right)_{x}}{u}+\frac{\left(\left(r^{n-1}-\tilde{r}^{n-1}\right) \tilde{v}\right)_{x}}{u}-\frac{\left(\tilde{r}^{n-1} \tilde{v}\right)_{x} U}{u \tilde{u}}\right]_{x} \\
& +\beta\left(r^{n-1}-\tilde{r}^{n-1}\right)\left[\frac{\left(\tilde{r}^{n-1} \tilde{v}\right)_{x}}{\tilde{u}}\right]_{x}-R r^{n-1}\left[\frac{\Theta}{u}-\frac{\tilde{\theta} U}{u \tilde{u}}\right]_{x} \\
& -R\left(r^{n-1}-\tilde{r}^{n-1}\right)\left[\frac{\tilde{\theta}}{\tilde{u}}-1\right]_{x}, \\
c_{V} \Theta_{t}= & \kappa_{Q}\left[\frac{r^{2 n-2} \Theta{ }_{x}}{u}+\frac{\left(r^{2 n-2}-\tilde{r}^{2 n-2}\right) \tilde{\theta}_{x}}{u}-\frac{\tilde{r}^{2 n-2} \tilde{\theta}{ }_{x} U}{u \tilde{u}}-\beta \frac{\left(r^{n-1} v\right)_{x}^{2} U}{u \tilde{u}}\right. \\
& +\beta \frac{\left[\left(\left(r^{n-1}-\tilde{r}^{n-1}\right) v\right)_{x}+\left(\tilde{r}^{n-1} V\right)_{x}\right]\left[\left(r^{n-1} v\right)_{x}+\left(\tilde{r}^{n-1} \tilde{v}\right)_{x}\right]}{\tilde{u}} \\
& -R \frac{\left(r^{n-1} v\right)_{x}\left[\Theta-\frac{\tilde{\theta} U}{\tilde{u}}\right]-R \frac{\tilde{\theta}}{\tilde{u}}\left\{\left(\left(r^{n-1}-\tilde{r}^{n-1}\right) v\right)_{x}+\left(\tilde{r}^{n-1} V\right)_{x}\right\}}{u} \\
& -2 \mu(n-1)\left[\left(r^{n-2}-\tilde{r}^{n-2}\right) v^{2}+\tilde{r}^{n-2}(v+\tilde{v}) V\right]_{x} \cdot
\end{aligned}
$$

If we integrate $\left[r^{n} / n-\tilde{r}^{n} / n\right]_{x}=U$ with respect to $x$, utilize (4.21), CauchySchwarz's inequality and (4.19), we obtain

$$
\begin{aligned}
& |r(x, t)-\tilde{r}(x, t)| \leqq C r^{-n+1}(x, t) \int_{0}^{x}|U| d y \\
& \quad \leqq C r^{-n+1}(x, t)\left(\int_{0}^{x} r^{2 n-2} d y\right)^{1 / 2}\left\|\left(r^{-n+1} U\right)(t)\right\| \leqq C \sqrt{x}\left\|\left(r^{-n+1} U\right)(t)\right\| \\
& \quad \leqq C r^{n / 2}(x, t)\left\|\left(r^{-n+1} U\right)(t)\right\|, \quad x \in[0, \infty), t \in[0, T]
\end{aligned}
$$

With the help of (3.18)-(3.19) for $r$ resp. $\tilde{r}$, (4.18), (4.21), and (4.25), one gets

$$
\begin{aligned}
& \left\|r^{-n+1}\left[\left(r^{n-1}-\tilde{r}^{n-1}\right) \tilde{v}\right]_{x}(t)\right\| \\
& \quad=\left\|r^{-n+1}\left[\left(r^{n-1}-\tilde{r}^{n-1}\right) \tilde{r}^{-n+1}\left(\tilde{r}^{n-1} \tilde{v}\right)\right]_{x}\right\| \\
& \quad \leqq C\left\|r^{-n+1}\left(\frac{u}{r}-\frac{\tilde{u}}{\tilde{r}}\right) \tilde{v}\right\|+C\left\|r^{-n}(r-\tilde{r}) \tilde{v}\right\|+C\left\|r^{-n}(r-\tilde{r})\left(\tilde{r}^{n-1} \tilde{v}\right)_{x}\right\|
\end{aligned}
$$




$$
\begin{aligned}
& \leqq C\left\{\left\|r^{-n}(r-\tilde{r}) \tilde{v}\right\|+\left\|r^{-n}(r-\tilde{r})\left(\tilde{r}^{n-1} \tilde{v}\right)_{x}\right\|+\left\|r^{-n+1} U\right\|\right\} \\
& \leqq C \max _{[0, \infty)}\left\{r^{-n}(\cdot, t)|r(\cdot, t)-\tilde{r}(\cdot, t)|\right\}\left(\|\tilde{v}\|+\left\|\left(\tilde{r}^{n-1} \tilde{v}\right)_{x}\right\|\right)+C\left\|r^{-n+1} U\right\| \\
& \leqq C\left\|\left(r^{-n+1} U\right)(t)\right\|\left\{1+\|\tilde{v}(t)\|+\left\|\tilde{u}_{t}(t)\right\|\right\} \\
& \leqq C\left\|\left(r^{-n+1} U\right)(t)\right\|, \quad 0 \leqq t \leqq T .
\end{aligned}
$$

Note that (4.26) still holds if $\tilde{v}$ resp. $\tilde{u}_{t}$ is replaced by $v$ resp. $u_{t}$.

Now multiply (4.22), (4.23) and (4.24) by $r^{-2 n+2} U, r^{-2 n+2} V$ and $r^{-2 n+2} \Theta$ respectively, and integrate then over $[0, \infty) \times[0, t](0 \leqq t \leqq T)$. Keeping in mind that $V, \Theta_{x}$ tend to zero for a.e. $t \in[0, T]$ as $x \rightarrow \infty$, we integrate by parts with respect to $x$, we use (3.18)-(3.19) for $r$ resp. $\tilde{r}$, (4.18), Cauchy-Schwarz's inequality, (4.21), (4.25), and (4.26) (as well as similar estimates like (4.26)) to arrive at

$$
\begin{aligned}
& \left\|\left(r^{-n+1} U, r^{-n+1} V, r^{-n+1} \Theta\right)(t)\right\|^{2}+\int_{0}^{t}\left(\left\|V_{x}\right\|^{2}+\left\|\Theta_{x}\right\|^{2}\right)(s) d s \\
& \leqq C \int_{0}^{t}\left\{1+\left\|\left(u_{t}, \tilde{u}_{t}, \tilde{r}^{n-1} \tilde{\theta}_{x}\right)(s)\right\|_{L^{\infty}}^{2}\right\}\left\|\left(r^{-n+1} U, r^{-n+1} V, r^{-n+1} \Theta\right)(s)\right\|^{2} d s \\
& \quad+C \int_{0}^{t} \max _{[0, \infty)}\left(r^{-2 n}|r-\tilde{r}|^{2}\right)(\cdot, s)\left\|\left(\tilde{u}-1, \tilde{\theta}-1, v, \tilde{r}^{n-1} \tilde{\theta}_{x}, \tilde{u}_{t}\right)(s)\right\|^{2} d s \\
& \leqq C \int_{0}^{t}\left\{1+\left\|\left(u_{t}, \tilde{u}_{t}, \tilde{r}^{n-1} \tilde{\theta}_{x}\right)(s)\right\|_{L^{\infty}}^{2}+\left\|\left(\tilde{u}-1, \tilde{\theta}-1, v, \tilde{r}^{n-1} \theta_{x}, \tilde{u}_{t}\right)(s)\right\|^{2}\right\} \\
& \quad \times\left\|\left(r^{-n+1} U, r^{-n+1} V, r^{-n+1} \Theta\right)(s)\right\|^{2} d s, \quad t \in[0, T] .
\end{aligned}
$$

Applying Gronwall's inequality to (4.27), taking (2.17) and (4.20) into account, we obtain $r^{-n+1} U=r^{-n+1} V=r^{-n+1} \Theta \equiv 0$, which implies $U=V=\Theta \equiv 0$ on $[0, \infty) \times[0, T]$. This proves the uniqueness.

III. Regularity. In the calculations that follow $C_{l}(l \in \mathbb{N})$ will denote a generic positive constant which may depend on $l$. Let $\left\{\tilde{u}_{k}, \tilde{v}_{k}, \tilde{\theta}_{k}\right\}$ denote the same subsequence as chosen in (4.6). If $u_{0}, v_{0}, \theta_{0}$ in addition satisfy (2.18), then by virtue of (3.17) in Theorem 3.3 we see that $\left\|\left(\left(\tilde{v}_{k}\right)_{t},\left(\tilde{\theta}_{k}\right)_{t},\left(\tilde{u}_{k}\right)_{x t},\left(\tilde{v}_{k}\right)_{x x},\left(\tilde{\theta}_{k}\right)_{x x}\right)\right\|_{L^{\infty}\left([0, T], L^{2}\right)}$ and $\left\|\left(\left(\tilde{u}_{k}\right)_{t t},\left(\tilde{v}_{k}\right)_{x t},\left(\tilde{\theta}_{k}\right)_{x t}\right)\right\|_{L^{2}\left(Q_{T}\right)}$ are uniformly bounded with respect to $k$. Hence we can extract a subsequence, still denoted by $\left\{\tilde{u}_{k}, \tilde{v}_{k}, \tilde{\theta}_{k}\right\}$, such that as $k \rightarrow \infty$,

$$
\begin{gathered}
\left(\left(\tilde{v}_{k}\right)_{t},\left(\tilde{\theta}_{k}\right)_{t},\left(\tilde{u}_{k}\right)_{x t},\left(\tilde{v}_{k}\right)_{x x},\left(\tilde{\theta}_{k}\right)_{x x}\right) \rightarrow\left(v_{t}, \theta_{t}, u_{x t}, v_{x x}, \theta_{x x}\right) \quad\left(\text { weak-*) in } L^{\infty}\left([0, T], L^{2}\right),\right. \\
\left(\left(\tilde{u}_{k}\right)_{t t},\left(\tilde{v}_{k}\right)_{x t},\left(\tilde{\theta}_{k}\right)_{x t}\right) \rightarrow\left(u_{t t}, v_{x t}, \theta_{x t}\right) \quad\left(\text { weakly) in } L^{2}\left(Q_{T}\right),\right.
\end{gathered}
$$

and

$$
\left\|\left(v_{t}, \theta_{t}, u_{x t}, v_{x x}, \theta_{x x}\right)\right\|_{L^{\infty}\left([0, T], L^{2}\right)}+\left\|\left(u_{t t}, v_{x t}, \theta_{x t}\right)\right\|_{L^{2}\left(Q_{T}\right)} \leqq C .
$$

Recalling (4.7) and (4.14), using (4.5), (4.8), and (4.29), we get

$$
\begin{gathered}
r, r_{t} r_{x} \in C^{0}([0, \infty) \times[0, T]), \quad r_{t t}, r_{x t}, r_{x x}, r_{x x t} \in L^{\infty}\left([0, T], L^{2}\right), \\
r_{x t t} \in L^{2}\left(Q_{T}\right) .
\end{gathered}
$$


Employing the estimates (4.5) and (4.29), now we can derive the bounds of the Hölder norm of $u, v, \theta, r$.

Lemma 4.1. For any $l \in \mathbb{N}$ we have

$$
\left\|\left(u, v, v_{x}, \theta, \theta_{x}, r, r_{x}\right)\right\|_{C^{1 / 3,1 / 6}\left(\bar{Q}_{T}^{l}\right)} \leqq C_{l} .
$$

Proof. On account of (4.5), (4.29) and Sobolev's imbedding theorem (cf. the proof of (4.8)), one has $u, v, v_{x}, \theta, \theta_{x} \in C^{0}\left(\bar{Q}_{T}\right)$. Moreover, $|u|,|v|,\left|v_{x}\right|,|\theta|,\left|\theta_{x}\right|$ are bounded (by a constant) on $[0, \infty) \times[0, T]$. From (4.30) we conclude that for any $l \in \mathbb{N}|r(x, t)|,\left|r_{x}(x, t)\right| \leqq C_{l}$ for all $(x, t) \in[0, l] \times[0, T]$. We use (4.5), (4.29) and Cauchy-Schwarz's inequality to see that for any $l \in \mathbb{N} \theta(x, t)$ resp. $\theta_{x}(x, t)$ is uniformly Hölder continuous in $t$ resp. in $x$ with exponent $1 / 2$ on $[0, l] \times[0, T]$ (also cf. [5], Lemma 3.7). A standard interpolation property (see [5, Lemma 3.3] or [15, Chapter II, Lemma 3.1]) implies that $\theta_{x}$ is also uniformly Hölder continuous in $t$ with exponent $1 / 6$ on $\bar{Q}_{T}^{l}$; hence $\left\|\theta_{x}\right\|_{C^{1 / 3,1 / 6}\left(\bar{Q}_{T}^{l}\right)} \leqq C_{l}$. This immediately yields $\|\theta\|_{C^{1 / 3,1 / 6}\left(\bar{Q}_{T}^{l}\right)} \leqq C_{l}$. Similarly, we can show $\left\|v_{x}\right\|_{C^{1 / 3,1 / 6}\left(\bar{Q}_{T}^{l}\right)} \leqq C_{l}$ and thereby $\|v\|_{C^{1 / 3,1 / 6}\left(\bar{Q}_{T}^{l}\right)} \leqq C_{l},\|u\|_{C^{1 / 3,1 / 6}\left(\bar{Q}_{T}^{l}\right)} \leqq C_{l}$. Therefore, from (4.7) and (4.14) we get $\left\|\left(r, r_{x}\right)\right\|_{C^{1 / 3,1 / 6}\left(\bar{Q}_{T}^{l}\right)} \leqq C_{l}$. This completes the proof.

Multiplying (2.11) with $r^{-n+1}$, integrating with respect to $t$ over $[0, t](t \in$ $[0, T])$, using (2.10) and noting that $\left[u_{t} / u\right]_{x}=\left[u_{x} / u\right]_{t}$ and $r^{-n+1} v_{t}=\left(r^{-n+1} v\right)_{t}+$ $(n-1) r^{-n} v^{2}$, we arrive at

$$
\begin{aligned}
u_{x}(x, t)= & u(x, t)\left(\frac{\left(u_{0}\right)_{x}}{u_{0}}+\frac{r^{-n+1} v(x, t)-r_{0}^{-n+1} v_{0}}{\beta}\right) \\
& +\frac{u(x, t)}{\beta} \int_{0}^{t}\left(R \frac{\theta_{x}}{u}+(n-1) \frac{v^{2}}{r^{n}}\right)(x, s) d s \\
& -\frac{R}{\beta} u(x, t) \int_{0}^{t} \frac{\theta(x, s) u_{x}(x, s)}{u^{2}(x, s)} d s, \quad x \in[0, \infty), t \in[0, T] .
\end{aligned}
$$

If we take the absolute value on both sides of (4.32) and apply Gronwall's inequality, we infer by (4.30), (4.9), (4.11), and (4.31) that for any $l \in \mathbb{N}$,

$$
\max _{[0, l] \times[0, T]}\left|u_{x}\right| \leqq C_{l} .
$$

Using an argument similar to the one used for (4.33) we obtain that for $\left(x_{0}, t_{0}\right) \in$ $[0, \infty) \times[0, T]\left|u_{x}(x, t)-u_{x}\left(x_{0}, t_{0}\right)\right| \leqq h\left(x_{0}, t_{0}, x, t\right) \rightarrow 0$ as $x \rightarrow x_{0}$ and $t \rightarrow t_{0}$, which gives $u_{x} \in C^{0}\left(\bar{Q}_{T}\right)$. Taking $\|\cdot\|_{C^{1 / 3,1 / 6}\left(\bar{Q}_{T}^{l}\right)}(l \in \mathbb{N})$ on both sides of (4.32), again applying Gronwall's inequality, and taking (4.31) and (4.33) into account, one gets

$$
\left\|u_{x}\right\|_{C^{1 / 3,1 / 6}\left(\bar{Q}_{T}^{l}\right)} \leqq C_{l} \quad(l \in \mathbb{N})
$$

which, with the help of (4.14) and (4.31), leads to

$$
\left\|r_{x x}\right\|_{C^{1 / 3,1 / 6}\left(\bar{Q}_{T}^{l}\right)} \leqq C_{l} \quad(l \in \mathbb{N}) .
$$


Let $2 l \in \mathbb{N}$ be arbitrary but fixed, let $\phi_{2 l}(x)$ be the same as in Sect. 3. We multiply Eqs. (2.10) and (2.11) by $\phi_{2 l}$ to obtain

$$
\begin{aligned}
&\left(\phi_{2 l} v\right)_{t}-\beta r^{n-1}\left[\frac{\left(r^{n-1} \phi_{2 l} v\right)_{x}}{u}\right]_{x}=-R r^{n-1}\left[\frac{\theta}{u}\right]_{x} \phi_{2 l}-\beta r^{n-1}\left[\frac{r^{n-1} v\left(\phi_{2 l}\right)_{x}}{u}\right]_{x} \\
&-\beta r^{n-1} \frac{\left(r^{n-1} v\right)_{x}\left(\phi_{2 l}\right)_{x}}{u} \\
& c_{V}\left(\phi_{2 l} \theta\right)_{t}-\kappa_{Q}\left[\frac{r^{2 n-2}\left(\phi_{2 l} \theta\right)_{x}}{u}\right]_{x}=-\kappa_{Q}\left[\frac{r^{2 n-2} \theta\left(\phi_{2 l}\right)_{x}}{u}\right]_{x}-\kappa_{Q} \frac{r^{2 n-2} \theta_{x}\left(\phi_{2 l}\right)_{x}}{u} \\
&-\frac{1}{u}\left[\beta\left(r^{n-1} v\right)_{x}-R \theta\right]\left(r^{n-1} v\right)_{x} \phi_{2 l}+2 \mu(n-1)\left(r^{n-2} v^{2}\right)_{x} \phi_{2 l} .
\end{aligned}
$$

It is easy to see by the definition of $\phi_{2 l}(x)$ and (2.14) that

$$
\left(\phi_{2 l} v\right)(0, t)=\left(\phi_{2 l} v\right)(2 l, t)=0, \quad\left(\phi_{2 l} \theta\right)_{x}(0, t)=\left(\phi_{2 l} \theta\right)_{x}(2 l, t)=0, t \geqq 0 .
$$

Thus we may consider (4.36)-(4.37) as the linear parabolic equations (for $\phi_{2 l} v$ and $\left.\phi_{2 l} \theta\right)$ with the boundary conditions $(4.38)$ on $(0,2 l) \times(0, T)$. Moreover, by virtue of (4.31) and (4.34)-(4.35), the coefficients and the right-hand sides of Eqs. (4.36)-(4.37) are Hölder-continuous on $[0,2 l] \times[0, T]$ with exponents $1 / 3$ in $x$ and $1 / 6$ in $t$. By the classical Schauder-Friedman estimates $[15,6]$ we infer $\phi_{2 l} v$, $\phi_{2 l} \theta \in C^{2+\beta, 1+\beta / 2}([0,2 l] \times[0, T])$ with $\beta:=\min \{\alpha, 1 / 3\}$, which gives

$$
v, \quad \theta \in C^{2+\beta, 1+\beta / 2}([0, l] \times[0, T]),
$$

since $\phi_{l}(x)=1$ for $x \leqq l$. Equation (4.39) together with (2.10), (4.31) and (4.34) implies

$$
u, u_{t} \in C^{1+\beta,(1+\beta) / 2}([0, l] \times[0, T]) .
$$

Equations (4.39) and (4.40) imply that $u, v, v_{x}, \theta, \theta_{x}, r, r_{x}$ in $C^{1,1 / 2}\left(\bar{Q}_{T}^{l}\right)$. With the help of this fact we can repeat the same procedure as used for $(4.34)-(4.40)$ to obtain the assertions (4.39)-(4.40) with $\beta=\min \{\alpha, 1\} \equiv \alpha$. Since $l \in \mathbb{N}$ is arbitrary, from (4.39)-(4.40) we get (2.19). The proof of Theorem 2.1 is complete.

\section{Large-Time Behavior in the Case of $n=3$}

In this section we show for $n=3$ that as $t \rightarrow \infty, v$ goes to zero in $L^{2 j}$ for $j \geqq 2$ arbitrary but fixed, and $u$ remains uniformly bounded for all $t \geqq 0$. Throughout this section the same letter $\Lambda$ will denote various positive constants which are in particular independent of $t, k$ and $x$. We will use the same notation as in Sect. 4. We have the following large-time behavior.

Theorem 5.1. Let $n=3$ and $\{u, v, \theta\}$ be the generalized solution established in Theorem 2.1. Assume $3 \lambda+2 \mu>0$. Then

i) There are positive constants $\gamma_{1}, \gamma_{2}$ independent of $t$ and $x$, such that $\gamma_{1} \leqq$ $u(x, t) \leqq \gamma_{2}$ for all $x, t \geqq 0$.

ii) For an arbitrary but fixed integer $j \geqq 2,\|v(t)\|_{L^{2 J}} \rightarrow 0$ as $t \rightarrow \infty$. 
Proof. Let $\left\{u_{k}, v_{k}, \theta_{k}\right\}$ be the same as in Sect. 3. First we adapt and modify an idea of Kazhikhov [12] (also cf. [3]) for the one-dimensional case in bounded intervals to give a representation for $u_{k}$.

$$
\begin{aligned}
& \text { Let } \\
& \Phi_{k}(x, t):=\int_{0}^{t} \sigma(x, s) d s+\int_{0}^{x}\left\{\left(r_{k}^{0}\right)^{-2} v_{k}^{0}\right\}(y) d y+2 \int_{0}^{t} \int_{x}^{k}\left\{r_{k}^{-3} v_{k}^{2}\right\}(y, s) d y d s,
\end{aligned}
$$

where $\sigma$ is defined by (3.40) in Sect. 3. Note that in view of (3.18) $\Phi_{k}$ satisfies

$$
\partial_{t} \Phi_{k}=\beta \frac{\left(r_{k}^{2} v_{k}\right)_{x}}{u_{k}}-R \frac{\theta_{k}}{u_{k}}+\frac{2}{3} \frac{\left(r_{k}^{3}\right)_{x}}{u_{k}} \int_{x}^{k} r_{k}^{-3} v_{k}^{2} d y
$$

By virtue of (3.4), a partial integration in the variable $t$ and (3.18), $\partial_{x} \Phi_{k}=r_{k}^{-2} v_{k}$. So, multiplying (5.2) by $u_{k}$ and using (3.3), we arrive at

$$
\left(u_{k} \Phi_{k}\right)_{t}-\left(r_{k}^{2} v_{k} \Phi_{k}\right)_{x}=-\frac{v_{k}^{2}}{3}-R \theta_{k}+\beta\left(r_{k}^{2} v_{k}\right)_{x}+\frac{2}{3}\left[r_{k}^{3} \int_{x}^{k} r_{k}^{-3} v_{k}^{2} d y\right]_{x} .
$$

Keeping in mind that $v_{k}$ vanishes on the boundary and $r_{k}(0, t)=a$, we integrate the above identity over $[0, k] \times[0, t]$ to infer

$$
\begin{aligned}
\int_{0}^{k}\left(u_{k} \Phi_{k}\right)(x, t) d x= & \int_{0}^{k}\left(u_{k}^{0} \Phi_{k}^{0}\right)(x) d x-\int_{0}^{t} \int_{0}^{k}\left(\frac{v_{k}^{2}}{3}+R \theta_{k}\right) d x d s \\
& -\frac{2 a^{3}}{3} \int_{0}^{t} \int_{0}^{k} r_{k}^{-3} v_{k}^{2} d x d s,
\end{aligned}
$$

where $\Phi_{k}^{0}(x):=\Phi_{k}(x, 0)$. It follows from integration of (3.3) over $[0, k] \times[0, t]$ and use of (3.7) that $\int_{0}^{k} u_{k}(x, t) d x=\int_{0}^{k} u_{k}^{0}(x) d x=: u_{k}^{*}$ for $t \geqq 0$. Moreover, by virtue of Lemma $3.1 u_{k}^{*}$ satisfies

$$
\begin{aligned}
& k / 2 \leqq k-\sqrt{k}\left\|u_{k}^{0}-1\right\| \leqq u_{k}^{*} \leqq k+\sqrt{k}\left\|u_{k}^{0}-1\right\| \leqq 2 k \\
& \quad \text { for some } k_{0}, \text { all } k \geqq k_{0} \text { and } t \geqq 0 .
\end{aligned}
$$

Note that $u_{k}>0$. If we apply the mean value theorem to (5.4), we conclude that for each $t \geqq 0$ there is an $x_{k}(t) \in[0, k]$ such that

$$
\Phi_{k}\left(x_{k}(t), t\right)=\frac{1}{u_{k}^{*}} \int_{0}^{k} \Phi_{k}(x, t) u_{k}(x, t) d x .
$$

Therefore from (5.1), (5.3) and (5.5) we get

$$
\begin{gathered}
\int_{0}^{t} \sigma_{k}\left(x_{k}(t), s\right) d s=\Phi_{k}\left(x_{k}(t), t\right)-\int_{0}^{x_{k}(t)}\left(r_{k}^{0}\right)^{-2} v_{k}^{0} d y-2 \int_{0}^{t} \int_{x_{k}(t)}^{k} r_{k}^{-3} v_{k}^{2} d y d s \\
=-\frac{1}{u_{k}^{*}} \int_{0}^{t} \int_{0}^{k}\left(\frac{v_{k}^{2}}{3}+R \theta_{k}\right) d x d s-\frac{2 a^{3}}{3 u_{k}^{*}} \int_{0}^{t} \int_{0}^{k} r_{k}^{-3} v_{k}^{2} d x d s \\
-2 \int_{0}^{t} \int_{x_{k}(t)}^{k} r_{k}^{-3} v_{k}^{2} d x d s+\frac{1}{u_{k}^{*}} \int_{0}^{k} u_{k}^{0} \Phi_{0} d x-\int_{0}^{x_{k}(t)}\left(r_{k}^{0}\right)^{-2} v_{k}^{0} d y, \quad t \geqq 0 .
\end{gathered}
$$


Using (5.6), we have the following representation:

$$
u_{k}(x, t)=\frac{D_{k}(x, t)}{B_{k}(x, t)}\left\{1+\frac{R}{\beta} \int_{0}^{t} \frac{\theta_{k}(x, s) B_{k}(x, s)}{D_{k}(x, s)} d s\right\}, \quad x \in[0, k], t \geqq 0,
$$

where

$$
\begin{aligned}
D_{k}(x, t): & =u_{k}^{0}(x) \exp \left\{\frac{1}{\beta}\left[\frac{1}{u_{k}^{*}} \int_{0}^{k} u_{k}^{0} \Phi_{0} d x-\int_{0}^{x}\left(r_{k}^{0}\right)^{-2} v_{k}^{0} d y+\int_{x_{k}(t)}^{x} r_{k}^{-2} v_{k} d y\right]\right\}, \\
B_{k}(x, t): & =\exp \left\{\frac { 1 } { \beta } \left[\frac{1}{u_{k}^{*}} \int_{0}^{t} \int_{0}^{k}\left(\frac{v_{k}^{2}}{3}+R \theta_{k}\right) d x d s+\frac{2 a^{3}}{3 u_{k}^{*}} \int_{0}^{t} \int_{0}^{k} r_{k}^{-3} v_{k}^{2} d y d x d s\right.\right. \\
& \left.\left.+2 \int_{0}^{t} \int_{x}^{k} r_{k}^{-3} v_{k}^{2} d y d s\right]\right\},
\end{aligned}
$$

and $x_{k}(t) \in[0, k]$ is the same as in (5.5).

In fact, note that (3.29) can be written in the form $r_{k}^{-2} \partial_{t} v_{k}=\partial_{x} \sigma_{k}=\left[\beta\left(\log u_{k}\right)_{t}\right.$ $\left.-R \theta_{k} / u_{k}\right]_{x}$. We integrate this over $[0, t]$ and then over $\left[x_{k}(t), x\right]$. If we integrate by parts with respect to $t$, utilize (3.18) and (5.6), we infer

$$
\begin{aligned}
& \beta \log u_{k}-R \int_{0}^{t} \frac{\theta_{k}}{u_{k}} d s=\beta \log u_{k}^{0}+\int_{0}^{t} \sigma_{k}\left(x_{k}(t), s\right) d s+\int_{x_{k}(t)}^{x} \int_{0}^{t} r_{k}^{-2}\left(v_{k}\right)_{t} d s d y \\
& =\beta \log u_{k}^{0}-\frac{1}{u_{k}^{*}} \int_{0}^{t} \int_{0}^{k}\left(\frac{v_{k}^{2}}{3}+R \theta_{k}\right) d x d s-\frac{2 a^{3}}{3 u_{k}^{*}} \int_{0}^{t} \int_{0}^{k} r_{k}^{-3} v_{k}^{2} d x d s \\
& -2 \int_{0}^{t} \int_{x}^{k} r_{k}^{-3} v_{k}^{2} d y d s+\int_{x_{k}(t)}^{x} r_{k}^{-2} v_{k} d y+\frac{1}{u_{k}^{*}} \int_{0}^{k} u_{k}^{0} \Phi_{0} d x-\int_{0}^{x}\left(r_{k}^{0}\right)^{-2} v_{k}^{0} d y,
\end{aligned}
$$

which, when the exponentials are taken, turns into

$$
\frac{B_{k}(x, t)}{D_{k}(x, t)}=\frac{1}{u_{k}(x, t)} \exp \left(\frac{R}{\beta} \int_{0}^{t} \frac{\theta_{k}(x, s)}{u_{k}(x, s)} d s\right) .
$$

Multiplying (5.8) by $R \theta_{k} / \beta$ and integrating over [0,t], we find that

$$
\exp \left(\frac{R}{\beta} \int_{0}^{t} \frac{\theta_{k}(x, s)}{u_{k}(x, s)} d s\right)=1+\frac{R}{\beta} \int_{0}^{t} \frac{\theta_{k}(x, s) B_{k}(x, s)}{D_{k}(x, s)} d s .
$$

Substituting this into (5.8), we obtain the (5.7).

Next we derive uniform bounds on $u_{k}(x, t)$ by using the representation (5.7). Integration of the second identity in (3.18) over $[0, x]$ and use of (3.26) lead to

$$
r_{k}^{3}(x, t)=a^{3}+3 \int_{0}^{x} u_{k}(y, t) d y \geqq a^{3}+3 \sum_{i=0}^{[x]} \int_{i=1}^{i} u_{k} d y \geqq \Lambda(2+[x]) \geqq \Lambda(1+x)
$$


for all $x \in[0, k]$ and $t \geqq 0$. Recalling (5.1), we get from Cauchy-Schwarz's inequality, (5.9), (3.20) and (5.4) that

$$
\begin{aligned}
& \left|\int_{x_{k}(t)}^{x} r_{k}^{-2} v_{k} d y\right|,\left|\int_{0}^{x}\left(r_{k}^{0}\right)^{-2} v_{k}^{0} d y\right|, \frac{1}{u_{k}^{*}}\left|\int_{0}^{k} u_{k}^{0} \Phi_{k}^{0} d x\right| \\
& \quad \leqq C\left(\int_{0}^{k}(1+x)^{-4 / 3} d x\right)^{1 / 2} \times\left(\left\|v_{k}^{0}\right\|+\left\|v_{k}(t)\right\|\right)+\left\|\Phi_{k}^{0}\right\|_{L^{\infty}}\left(u_{k}^{*}\right)^{-1}\left(k+\sqrt{k}\left\|u_{0}-1\right\|\right) \\
& \leqq \Lambda \quad \forall x \in[0, k]
\end{aligned}
$$

which, in view of the definition of $D_{k}(x, t)$, leads to

$$
0<\Lambda^{-1} \leqq D_{k}(x, t) \leqq \Lambda \quad \forall x \in[0, k], t \geqq 0
$$

By (3.19), (3.26) and (5.4) we find

$$
\frac{B_{k}(x, s)}{B_{k}(x, t)} \leqq \exp \left\{-\frac{R}{\beta u_{k}^{*}} \int_{s}^{t} \int_{0}^{k} \theta_{k}(x, s) d x d s\right\} \leqq \exp \left\{-\frac{R \alpha_{1}(t-s)}{2 \beta}\right\}, \quad t \geqq s \geqq 0 .
$$

Similarly,

$$
\frac{B_{k}(x, s)}{B_{k}(x, t)} \geqq \Lambda e^{-\Lambda_{1}(t-s)}, \quad t \geqq s \geqq 0 ; \quad e^{\Lambda t} \geqq B_{k}(x, t) \geqq 1, \quad t \geqq 0
$$

with $\Lambda_{1}$ being independent of $t$ and $k$, where we have used (3.19), (3.20) and (3.26).

It is easy to see by (3.38) and (3.27) that

$$
\begin{aligned}
& \frac{\alpha_{1}}{2}-\Lambda \max _{[0, k]} u_{k}(\cdot, t) \int_{0}^{k} \frac{\left(\theta_{k}\right)_{x}^{2}}{u_{k} \theta_{k}^{2}} d x \leqq \theta_{k}(x, t) \\
& \quad \leqq 2 \alpha_{2}+\Lambda \max _{[0, k]} u_{k}(\cdot, t) \int_{0}^{k} \frac{\left(\theta_{k}\right)_{x}^{2}}{u_{k} \theta_{k}^{2}} d x, \quad x \in[0, k], t \geqq 0 .
\end{aligned}
$$

Hence it follows from (5.7), (5.10), (5.11), (5.13) that for all $x \in[0, k], t \geqq 0$,

$$
\begin{aligned}
u_{k}(x, t) & \leqq \Lambda+\Lambda \int_{0}^{t}\left(1+\max _{[0, k]} u_{k} \int_{0}^{k} \frac{\left(\theta_{k}\right)_{x}^{2}}{u_{k} \theta_{k}^{2}} d x\right) e^{-\Lambda^{-1}(t-s)} d s \\
& \leqq \Lambda+\Lambda \int_{0}^{t} \max _{[0, k]} u_{k} \int_{0}^{k} \frac{\left(\theta_{k}\right)_{x}^{2}}{u_{k} \theta_{k}^{2}} d x d s .
\end{aligned}
$$

Applying Gronwall's inequality to the above inequality and utilizing (3.20), one obtains $u_{k}(x, t) \leqq \gamma_{2}$ for all $x \in[0, k]$ and $t \geqq 0$, where $\gamma_{2}$ is a positive constant independent of $t, k$ and $x$. Therefore we make use of (5.7), (5.10), (5.12), and 
(5.13) to infer

$$
\begin{aligned}
u_{k}(x, t) & \geqq \Lambda \int_{0}^{t} \frac{B_{k}(x, s)}{B_{k}(x, t)} \theta_{k}(x, s) d s \geqq \Lambda_{2} \int_{0}^{t}\left(\frac{\alpha_{1}}{2}-\Lambda \max _{[0, k]} u_{k} \int_{0}^{k} \frac{\left(\theta_{k}\right)_{x}^{2}}{u_{k} \theta_{k}^{2}} d x\right) e^{-\Lambda_{1}(t-s)} d s \\
& \geqq \frac{\Lambda_{2} \alpha_{1}}{2} \int_{0}^{t} e^{-\Lambda_{1}(t-s)} d s-\Lambda_{2} \Lambda \gamma_{2} \int_{0}^{t} \int_{0}^{k} \frac{\left(\theta_{k}\right)_{x}^{2}}{u_{k} \theta_{k}^{2}} e^{-\Lambda_{1}(t-s)} d x d s \\
& \geqq \frac{\Lambda_{2} \alpha_{1}}{2 \Lambda_{1}}\left(1-e^{-\Lambda_{1} t}\right)-\Lambda e^{-\Lambda_{1} t / 2} \int_{0}^{t / 2} \int_{0}^{k} \frac{\left(\theta_{k}\right)_{x}^{2}}{u_{k} \theta_{k}^{2}} d x d s-\Lambda \int_{t / 2}^{t} \int_{0}^{k} \frac{\left(\theta_{k}\right)_{x}^{2}}{u_{k} \theta_{k}^{2}} d x d s \\
& \geqq \frac{\Lambda_{2} \alpha_{1}}{4 \Lambda_{1}}>0
\end{aligned}
$$

for all $t \geqq T_{0}$ and some (large) $T_{0}>0$, where $\Lambda_{2}$ is independent of $t, k$ and $x$. Furthermore, from (5.7) and (5.10) we get $u_{k}(x, t) \geqq D_{k}(x, t) / B_{k}(x, t) \geqq e^{-\Lambda t} / \Lambda$ for all $x \in[0, k]$ and $t \geqq 0$. Combining this with (5.14) we see that there are positive constants $\gamma_{1}, \gamma_{2}$ independent of $t, k$ and $x$, such that $\gamma_{1} \leqq u_{k}(x, t) \leqq \gamma_{2}$ for all $x \in[0, k], t \geqq 0$. This combined with (4.2), (4.6) and (4.8) yields i) in Theorem 5.1 .

Note that by virtue of (5.9), (4.2), (4.4), (4.6) and (4.10), the estimates (3.20), (3.26) and (5.9) still hold when $u_{k}, v_{k}, \theta_{k}, r_{k},[0, k]$ are replaced by $u, v, \theta, r,[0, \infty)$, respectively. Thus it follows from $i$ ) of Theorem 5.1 that

$$
\begin{aligned}
\int_{0}^{t} \max _{x \geqq 0}\left[r v^{2}\right](x, s) d s \\
\quad=\int_{0}^{t} \max _{x \geqq 0} \frac{1}{r^{3}}\left(\int_{0}^{x}\left(r^{2} v\right)_{x} d y\right)^{2} d s \leqq \Lambda \int_{0}^{t} \max _{x \geqq 0} \frac{1}{r^{3}} \int_{0}^{x} \frac{\left(r^{2} v\right)_{x}^{2}}{u \theta} d y \int_{0}^{x} \theta d y d s \\
\quad \leqq \Lambda \int_{0}^{t} \max _{x \geqq 0} \frac{(1+x)}{r^{3}} \int_{0}^{x} \frac{\left(r^{2} v\right)_{x}^{2}}{u \theta} d y \leqq \Lambda \int_{0}^{t} \int_{0}^{\infty} \frac{\left(r^{2} v\right)_{x}^{2}}{u \theta} d y \leqq \Lambda, \quad t \geqq 0 .
\end{aligned}
$$

From (3.26), (4.6) and (4.8) we get $\alpha_{1} \leqq \int_{0}^{1} \theta(x, t) d x \leqq \alpha_{2}$, which gives $\alpha_{1} \leqq$ $\theta(c(t), t) \equiv \bar{\theta}(t) \leqq \alpha_{2}$ for $t \geqq 0$ and some $c(t) \in[0,1]$. It is easy to see by (5.9) that

$$
\begin{aligned}
\int_{0}^{t} \max _{[0, \infty)}[\theta-\bar{\theta}]^{2}(\cdot, s) d s & \leqq \Lambda \int_{0}^{t} \int_{0}^{\infty} \frac{r^{4} \theta_{x}^{2}}{u \theta^{2}} d x \int_{0}^{\infty} \frac{\theta^{2}}{r^{4}} d x d s \\
& \leqq \Lambda+\Lambda \int_{0}^{t} \int_{0}^{\infty} \frac{r^{4} \theta_{x}^{2}}{u \theta^{2}} d x \max _{[0, \infty)}[\theta-\bar{\theta}]^{2}(s) d s
\end{aligned}
$$

Applying Gronwall's inequality and (3.20) to the above inequality, one gets

$$
\int_{0}^{t} \max _{[0, \infty)}[\theta(\cdot, s)-\bar{\theta}(s)]^{2} d s \leqq \Lambda \quad \text { for all } t \geqq 0
$$

Using (4.18), (4.20), and the estimate $\left(r^{2} v^{2}\right)(x, t)=\int_{0}^{x}\left[\left(r^{2} v\right)_{x} v+r^{2} v v_{x}\right] \leqq$ $C \int_{0}^{\infty}\left(u_{t}^{2}+v^{2}\right) d x \leqq C$ that follows from (4.20) and (2.10), we deduce that for 
each $t \geqq 0, \int_{0}^{t}\left(\beta u_{t}-R \theta\right)\left(r^{2} v^{3}\right)(x, s) d s \rightarrow 0$ as $x \rightarrow \infty$. Therefore if we multiply (2.11) by $2 j v^{2 j-1}(j \geqq 2)$ in $L^{2}((0, \infty) \times(0, t))$, integrate by parts with respect to $x$, utilize (4.14) and $\mathrm{i})$ of Theorem 5.1 , we deduce

$$
\begin{aligned}
& \int_{0}^{\infty} v^{2 j}(x, t) d x+j^{2} \int_{0}^{t} \int_{0}^{\infty} v^{2 j-2}\left(r^{2} v\right)_{x}^{2} d x d s \\
& \quad \leqq \Lambda+\Lambda j^{2} \int_{0}^{t} \int_{0}^{\infty}\left\{v^{2 j}+\theta\left(\left|v^{2 j-2}\left(r^{2} v\right)_{x}\right|+r^{-1}\left|v^{2 j-1}\right|\right)\right\} d x d s \equiv \Lambda+I_{j}(t)
\end{aligned}
$$

Denote $F_{j}(t):=\max _{0 \leqq s \leqq t} \int_{0}^{\infty} v^{2 j}(x, s) d x$. Then $I_{j}$ can be estimated as follows, using $(3.20),(5.15)-(5.16)$,

$$
\begin{aligned}
I_{2}(t) \leqq & \Lambda+\Lambda \int_{0}^{t} \int_{0}^{\infty}\left(\left|\left(r^{2} v\right)_{x}\right| v^{2}+r^{-1} v^{3}\right)(|\theta-\bar{\theta}|+1) d x d s \\
\leqq & +\int_{0}^{t} \int_{0}^{\infty}\left\{\left[v^{2}+(u \theta)^{-1}\right]\left(r^{2} v\right)_{x}^{2}+\Lambda \theta v^{4}\right\} d x d s \\
& +\Lambda \int_{0}^{t} \max _{[0, \infty)}\left\{|\theta-\bar{\theta}|^{2}+r v^{2}\right\} \int_{0}^{\infty}\left(v^{2}+r^{-4}\right) d x d s \\
\leqq & +\int_{0}^{t} \int_{0}^{\infty} v^{2}\left(r^{2} v\right)_{x}^{2} d x d s+\Lambda \int_{0}^{t} \max _{[0, \infty)}|\theta-\bar{\theta}|^{2} \int_{0}^{\infty} v^{4} d x d s
\end{aligned}
$$

and for $j \geqq 3$,

$$
\begin{aligned}
I_{j}(t) \leqq & \Lambda j^{2} F_{j-1}(t)+\Lambda j^{2} \int_{0}^{t} \max _{[0, \infty)}\left\{|\theta-\bar{\theta}|^{2}+v^{2}\right\} \int_{0}^{\infty}\left(v^{2 j-2}+v^{2 j-4}\right) d x d s \\
& +\frac{j^{2}}{2} \int_{0}^{t} \int_{0}^{\infty} v^{2 j-2}\left(r^{2} v\right)_{x}^{2} d x d s \\
& \leqq \Lambda j^{2}\left(F_{j-1}+F_{j-2}\right)(t)+\frac{j^{2}}{2} \int_{0}^{t} \int_{0}^{\infty} v^{2 j-2}\left(r^{2} v\right)_{x}^{2} d x d s .
\end{aligned}
$$

Inserting (5.18)-(5.19) into (5.17), applying Gronwall's inequality and (5.16) if $j=2$, we arrive at

$$
F_{j}(t)+\int_{0}^{t} \int_{0}^{\infty} v^{2 j-2}\left(r^{2} v\right)_{x}^{2} d x d s \leqq \Lambda\left\{1+j^{2} F_{j-1}(t)+j(j-2) F_{j-2}(t)\right\}, \quad j \geqq 2,
$$

which by induction leads to

$$
\int_{0}^{\infty} v^{2 j}(x, t) d x+j^{2} \int_{0}^{t} \int_{0}^{\infty} v^{2 j-2}\left(r^{2} v\right)_{x}^{2} d x d s \leqq \Gamma_{j} \quad \forall t \geqq 0, j \geqq 2,
$$

where $\Gamma_{j}$ is a constant that depends only on $j$ and $\Lambda$. 
Using Eq. (2.11), (5.15)-(5.16), following the same arguments as used for (5.17)-(5.19), we get for $j \geqq 2$,

$$
\begin{aligned}
& \int_{0}^{\infty}\left|\frac{d}{d t} \int_{0}^{\infty} v^{2 j}(x, t) d x\right| d s \leqq \Lambda+\Lambda j^{2} \int_{0}^{\infty} \int_{0}^{\infty} v^{2 j-2}\left(r^{2} v\right)_{x}^{2} d x d s \\
& +\Lambda(j-2) j \max _{t \geqq 0} \int_{0}^{\infty}\left(v^{2 j-2}+v^{2 j-4}\right) d x<\infty,
\end{aligned}
$$

which together with (5.15) and (5.20) yields ii) of Theorem 5.1. The proof is complete.

\section{References}

1. Adams, R.A.: Sobolev Spaces. New York: Academic Press, 1975

2. Alikakos, N.D.: An application of the invariance principle to reaction-diffusion equations. J. Diff. Equations 33, 201-225 (1979)

3. Antontsev, S.N., Kazhikhov, A.V., Monakhov, V.N.: Boundary Value Problems in Mechanics of Nonhomogeneous Fluids. Amsterdam, New York: North-Holland, 1990

4. Batchelor, G.K.: An Introduction to Fluid Dynamics. London: Cambridge Univ. Press, 1967

5. Dafermos, C.M., Hsiao, L.: Global smooth thermomechanical processes in one-dimensional nonlinear thermoviscoelasticity. Nonlinear Anal. T.M.A. 6, 435-454 (1982)

6. Friedman, A.: Partial Differential Equations of Parabolic Type. Englewood Cliffs, NJ: PrenticeHall, 1964

7. Jiang, S.: On initial boundary value problems for a viscous, heat-conducting, one-dimensional real gas. J. Diff. Equations 110, 157-181 (1994)

8. Jiang, S.: On the asymptotic behavior of the motion of a viscous, heat-conducting, onedimensional real gas. Math. Z. 216, 317-336 (1994)

9. Jiang, S.: Remarks on the global existence in the dynamics of a viscous, heat-conducting, one-dimensional gas. Proc. of the Workshop on Qualitative Aspects and Appl. of Nonlinear Evol. Eqns., H. Beirao da Veiga, Ta-Tsien Li (eds.), Singapore: World Scientific Publ., 1994, pp. $156-162$

10. Jiang, S.: Global smooth solutions to the equations of a viscous, heat-conducting, onedimensional gas with density-dependent viscosity (submitted)

11. Kawashima, S., Nishida, T.: Global solutions to the initial value problem for the equations of one-dimensional motion of viscous polytropic gases. J. Math. Kyoto Univ. 21, 825-837 (1981)

12. Kawohl, B.: Global existence of large solutions to initial boundary value problems for a viscous, heat-conducting, one-dimensional real gas. J. Diff. Equations 58, 76-103 (1985)

13. Kazhikhov, A.V.: To a theory of boundary value problems for equations of one-dimensional non-stationary motion of viscous heat-conduction gases. Boundary Value Problems for Hydrodynamical Equations. Inst. Hydrodynamics, Siberian Branch Akad., USSR, No. 50, 1981, pp. 37-62 (Russian)

14. Kazhikhov, A.V., Shelukhin, V.V.: Unique global solution with respect to time of initial boundary value problems for one-dimensional equations of a viscous gas. J. Appl. Math. Mech. 41, 273-282 (1977)

15. Ladyzenskaja, O.A., Solonnikov, V.A., Ural'ceva, N.N.: Linear and Quasilinear Equations of Parabolic Type. Providence, Rhode Island: AMS, 1968

16. Matsumura, A., Nishida, T.: The initial value problem for the equations of motion of compressible viscous and heat-conductive fluids. Proc. Japan Acad. Ser. A 55, 337-342 (1979)

17. Matsumura, A., Nishida, T.: The initial value problem for the equations of motion of viscous and heat-conductive gases. J. Math. Kyoto Univ. 20, 67-104 (1980)

18. Matsumura, A., Nishida, T.: Initial boundary value problems for the equations of motion of general fluids. Computing Meth. in Appl. Sci. and Engin. V, R. Glowinski, J.L. Lions (eds.), Amsterdam: North-Holland, 1982, pp. 389-406 
19. Matsumura, A., Nishida, T.: Initial boundary value problems for the equations of motion of compressible viscous and heat-conductive fluids. Commun. Math. Phys. 89, 445-464 (1983)

20. Nagasawa, T.: On the one-dimensional motion of the polytropic ideal gas non-fixed on the boundary. J. Diff. Equations 65, 49-67 (1986)

21. Nagasawa, T.: On the outer pressure problem of the one-dimensional polytropic ideal gas. Japan J. Appl. Math. 5, 53-85 (1988)

22. Nagasawa, T.: On the one-dimensional free boundary problem for the heat-conductive compressible viscous gas. Lecture Notes in Num. Appl. Anal., M. Mimura, T. Nishida (eds.) Vol. 10, Tokyo: Kinokuniya/North-Holland, 1989, pp. 83-99

23. Nikolaev, V.B.: On the solvability of mixed problem for one-dimensional axisymmetrical viscous gas flow. Dinamicheskie zadachi Mekhaniki sploshnoj sredy, 63 Sibirsk. Otd. Acad. Nauk SSSR, Inst. Gidrodinamiki, 1983 (Russian)

24. Renardy, M., Hrusa, W.J., Nohel, J.A.: Mathematical Problems in Viscoelasticity. Pitman Monographs and Surveys in Pure and Appl. Math. 35, Longman Sci. Tech., 1987

25. Serrin, J.: Mathematical principles of classical fluid mechanics. Handbuch der Physik VIII/1, Berlin, Heidelberg, New York: Springer 1972, pp. 125-262

26. Valli, A.: Mathematical results for compressible flows. Mathematical Topics in Fluid Mechanics, J.F. Rodrigues, A. Sequeira (eds.) Pitman Research Notes in Math. Ser. 274, New York: John Wiley 1992, pp. 193-229

27. Valli, A., Zajączkowski, W.M.: Navier-Stokes Equations for compressible fluids: global existence and qualitative properties of the solutions in the general case. Commun. Math. Phys. 103, 259-296 (1986)

28. Yashima, H.F., Benabidallah, R.: Equation à symétrie sphérique d'un gaz visqueux et calorifère avec la surface libre. Annali Mat. Pura Applicata CLXVIII, 75-117 (1995)

29. Yashima, H.F., Benabidallah, R.: Unicite' de la solution de l'équation monodimensionnelle ou à symétrie sphérique d'un gaz visqueux et calorifère. Rendi. del Circolo Mat. di Palermo, Ser. II, XLII, 195-218 (1993)

Communicated by H. Araki 